\title{
Characterisation of microbiota in saliva, bronchoalveolar lavage fluid, non- malignant, peritumoural and tumour tissue in non-small cell lung cancer patients: a cross-sectional clinical trial
}

Rea Bingula ${ }^{1}$, Edith Filaire ${ }^{1,2}$, loana Molnar ${ }^{3,4,5}$, Eve Delmas ${ }^{6}$, Jean-Yves Berthon ${ }^{2}$, Marie-Paule Vasson ${ }^{1,7}$, Annick Bernalier-Donadille ${ }^{6}$ and Marc Filaire ${ }^{1,8^{*}}$ (I)

\begin{abstract}
Background: While well-characterised on its molecular base, non-small cell lung cancer (NSCLC) and its interaction with local microbiota remains scarcely explored. Moreover, current studies vary in source of lung microbiota, from bronchoalveolar lavage fluid (BAL) to tissue, introducing potentially differing results. Therefore, the objective of this study was to provide detailed characterisation of the oral and multi-source lung microbiota of direct interest in lung cancer research. Since lung tumours in lower lobes (LL) have been associated with decreased survival, characteristics of the microbiota in upper $(U L)$ and lower tumour lobes have also been examined.

Methods: Using 16S rRNA gene sequencing technology, we analysed microbiota in saliva, BAL (obtained directly on excised lobe), non-malignant, peritumoural and tumour tissue from 18 NSCLC patients eligible for surgical treatment. Detailed taxonomy, diversity and core members were provided for each microbiota, with analysis of differential abundance on all taxonomical levels (zero-inflated binomial general linear model with BenjaminiHochberg correction), between samples and lobe locations.

(Continued on next page)
\end{abstract}

\footnotetext{
* Correspondence: marc.filaire@clermont.unicancer.fr

'Université Clermont Auvergne, INRAE, UNH, F-63000 Clermont-Ferrand, France

${ }^{8}$ Thoracic Surgery Department, Centre Jean Perrin, 63011 Clermont-Ferrand, France

Full list of author information is available at the end of the article
}

C C The Author(s). 2020 Open Access This article is licensed under a Creative Commons Attribution 4.0 International License, which permits use, sharing, adaptation, distribution and reproduction in any medium or format, as long as you give appropriate credit to the original author(s) and the source, provide a link to the Creative Commons licence, and indicate if changes were made. The images or other third party material in this article are included in the article's Creative Commons licence, unless indicated otherwise in a credit line to the material. If material is not included in the article's Creative Commons licence and your intended use is not permitted by statutory regulation or exceeds the permitted use, you will need to obtain permission directly from the copyright holder. To view a copy of this licence, visit http://creativecommons.org/licenses/by/4.0/ The Creative Commons Public Domain Dedication waiver (http://creativecommons.org/publicdomain/zero/1.0/) applies to the data made available in this article, unless otherwise stated in a credit line to the data. 
(Continued from previous page)

Results: Diversity and differential abundance analysis showed clear separation of oral and lung microbiota, but more importantly, of BAL and lung tissue microbiota. Phylum Proteobacteria dominated tissue samples, while Firmicutes was more abundant in BAL and saliva (with class Clostridia and Bacilli, respectively). However, all samples showed increased abundance of phylum Firmicutes in LL, with decrease in Proteobacteria. Also, clades Actinobacteria and Flavobacteriia showed inverse abundance between BAL and extratumoural tissues depending on the lobe location. While tumour microbiota seemed the least affected by location, peritumoural tissue showed the highest susceptibility with markedly increased similarity to BAL microbiota in UL. Differences between the three lung tissues were however very limited.

Conclusions: Our results confirm that BAL harbours unique lung microbiota and emphasise the importance of the sample choice for lung microbiota analysis. Further, limited differences between the tissues indicate that different local tumour-related factors, such as tumour type, stage or associated immunity, might be the ones responsible for microbiota-shaping effect. Finally, the "shift" towards Firmicutes in LL might be a sign of increased pathogenicity, as suggested in similar malignancies, and connected to worse prognosis of the LL tumours.

Trial registration: ClinicalTrials.gov ID: NCT03068663. Registered February 27, 2017.

Keywords: Lung cancer, Lung microbiota, Non-small cell lung cancer, Bronchoalveolar lavage, Peritumoural lung tissue, Non-malignant lung tissue, Lower lobe tumour, Lobe location, Saliva

\section{Background}

Despite the advancements in its detection and treatment, lung cancer (LC) is still the leading cause of death by cancer worldwide [1]. Non-small cell lung cancer (NSCLC) is diagnosed in $85-90 \%$ of LC cases and presents the most frequent type of lung cancer. Unlike small cell lung cancer, NSCLC is operable in $20-25 \%$ of cases. This concerns mostly early stage tumours (stage I and II), sometimes locally advanced disease (stage III) and rarely oligometastatic disease (stage IV). Other treatments, such as chemotherapy, radiotherapy and until recently immunotherapy, are often associated with surgery as multimodality treatment. Even though surgery is recognised as the most effective initial treatment of NSCLC, the 5-year survival rates remain however low ( 90\% for stage IA 1 , and $\sim 12 \%$ for stage IIIC) $[2,3]$. Therefore, the tumour staging is used as the important prognostic tool based on tumour size, lymph node invasion and metastatic status [2]. Curiously, tumour lobe location has also been associated to tumours' aggressiveness, with tumours in lower lobes (LL) showing worse term and 5-year survival after resection than the ones in upper lobes (UL), still without a clear explanation [4-6].

Increasing interest in the interaction between host and its microbiota revealed its potential implication in health and disease, but also in tumour immunology and physiology [7-11]. Certain bacteria, such as genus Bifidobacterium [8] or species Enterococcus hirae [10], have been found to improve the efficiency of chemotherapy or immune-checkpoint inhibitors if administered orally in animal models. This phenomenon has been explained by their translocation from the gut to mesenteric lymph nodes, the priming of the upstream regulatory immune cells, such as dendritic cells, and causing increased reactivity against tumour epitopes [12, 13]. Moreover, administration of Bifidobacterium cocktail alone has been proved equally effective as the anti-PD-1 (Programmed cell Death protein 1) antibody in abolishing tumour growth in the animal melanoma model [8]. Finally, faecal transplantation from the patients responding (enriched in Bifidobacterium, Akkermansia, Faecalibacterium) or not (enriched in Bacteroidales) to the anti-cancer treatment has induced the same kind of response in receiving tumour-bearing animals $[8,14]$. These findings have been one of the most elucidating in terms of interactions between the host's immune system, gut microbiota and cancer.

Unlike local and systemic effects of the gut microbiota, the lung microbiota and its effects remain scarcely explored, being only recently accepted as one of the resident microbiota (and not only present during infection) $[15,16]$. Since then, an emerging number of studies turned to its exploration, notably in the context of cystic fibrosis, asthma and chronic obstructive pulmonary disease (COPD), interstitial lung disease, and lung transplantation [17-22]. Despite its impact on global cancerrelated death, lung cancer studies were surprisingly few and started to emerge only a few years ago. However, they confirmed that lung microbiota interacts with local immunity and modifies tumour properties. The microbial dysbiosis in antibiotic-treated or germ-free animals influenced growth of injected lung tumour cells [23, 24] while usage of penicillin, cephalosporins, or macrolides showed increasing risk of lung cancer in human subjects [25]. In lung cancer patients, lung microbiota from bronchoalveolar lavage fluid (BAL) enriched with supraglottic taxa was associated with pro-inflammatory profile and 
stimulation of Th17 cells with protumourigenic effect [26-28], and also exhibited different abundance and metabolic profiles compared to those of healthy subjects $[29,30]$. Interestingly, salivary microbiota was also found to show cancer specific profile, with genera Veillonella and Capnocytophaga more abundant in saliva of lung cancer patients [31]. At the present, only two studies analysed lung tissue microbiota in lung cancer. One found increased alpha diversity in non-malignant tissue compared to tumours as well as in adenocarcinoma compared to squamous cell carcinoma [32], while the other showed association between increased diversity of the non-malignant tissue (but not tumour) and decreased recurrence-free and disease-free survival [33]. Among studies on lung microbiota, those on BAL are the most numerous, since it remains the sample with acceptable ratio of contamination risk by upper airways, precision in lung microbiota sampling and invasiveness. However, this has been a potential source of contradictory information since varying characteristics of BAL and tissue microbiota, as a result of samples' different nature, have been previously suggested [21]. Therefore, there has been an increasing necessity to characterise the ground differences between different lung microbiota in NSCLC patients to enable better comprehension of the obtained results depending on the initial lung sample.

As its primary objective, this cross-sectional pilot study analysed lung microbiota from four different samples in 18 NSCLC patients eligible for surgery without neoadjuvant therapy. Lung microbiota was analysed in BAL, nonmalignant tissue, peritumoural tissue and tumour, as each sample should have different architectural and physiological characteristics. Unlike what was previously seen, in this study BAL was obtained directly from the excised lobe without passing through the upper airways to decrease the contamination risk. In addition to lung microbiota, salivary microbiota was characterised for each patient and used as an extra-pulmonary sample to put in perspective the relation with and between lung samples. As a second objective, we investigated whether tumour location in the UL or LL yields significant changes in these microbiota.

\section{Methods and patients}

\section{Patient recruitment and study design}

All patients were enrolled in a prospective study, approved by the CPP Sud Est VI Ethics Committee and registered at ClinicalTrials.gov (NCT03068663) [34]. Written informed consent was obtained from all patients before enrolment in the study and any study procedure.

Patients diagnosed with primary NSCLC eligible for surgical treatment with or without neoadjuvant therapy and presented before the Thoracic Oncologic Committee of the Centre Jean Perrin (Clermont-Ferrand, France) were preconsidered for inclusion to the study. Inclusion criteria were: age between 18 and 80 years, body mass index $(\mathrm{BMI})<29.9$, no antibiotics, corticoids, immunosuppressive drugs or having undergone pulmonary infections for at least the past 2 months, as well as no previous airway surgery or cancer treatment. Only patients included in the group of patients eligible for surgery without chemotherapy were taken into account in this manuscript.

At inclusion, patients received the tube for saliva collection and were asked to bring it with them the day of their hospital admission for surgery. Sampling of the lung was performed during the surgery immediately after excision of the tumour lobe, representing no additional inconvenience for patients apart from this standard medical procedure. Detailed inclusion/exclusion criteria, the study flowchart as well as detailed design and power calculation were previously published [34].

\section{Sampling \\ Saliva}

Saliva was collected as previously described [34]. Upon reception by the study personnel, the sample was stored at $-80^{\circ} \mathrm{C}$ until DNA extraction.

\section{Lung tissue and $B A L$}

Sampling of lung tissue and BAL during surgery was performed immediately after partial or complete pneumonectomy. The removed lung or lung lobe was placed in a sterile vessel and the tumour position was determined by palpation. First, a piece of non-malignant lung distal to the tumour (opposite side of the lobe) with an average size of 1 $\mathrm{cm}^{3}$ was clamped. The clamp was left in place until the end of the following procedure. Using a sterile syringe, the excised lung was inflated through the main bronchus. Bronchoalveolar lavage was performed by instilling $2 \times 40 \mathrm{~mL}$ of sterile physiological saline into the bronchus. After each instillation, the maximum amount of liquid inside the bronchus was retrieved $(8-10 \mathrm{~mL}$ in total) into $50 \mathrm{~mL}$ tube (designated as "BAL"). Then, the clamped wedge of nonmalignant tissue was cut off and designated as LUNG.DP ("distal piece"). Further, a pie-slice of the tumour (crosssection) was excised with its peritumoural tissue, after which the two were separated based on macroscopic histological difference. Tumour tissue sample was designated as "LUNG.T" and peritumoural tissue sample as "LUNG.PT". The tissues were snap-frozen in liquid nitrogen and then placed at $-80^{\circ} \mathrm{C}$ for long-term storage until DNA extraction. BAL was stored directly at $-80^{\circ} \mathrm{C}$.

\section{DNA extraction and negative controls}

DNA was extracted as previously described [34]. Saliva and BAL (cellular BAL) volume used for DNA extraction were 1 and $5 \mathrm{~mL}$, respectively. Initial tissue weights ranged from $377 \pm 236 \mathrm{mg}$ for LUNG.PT, to $1.441 \pm 1.016 \mathrm{~g}$ and $1.346 \pm 0.899 \mathrm{~g}$ for LUNG.DP and LUNG.T. Even though 
the initial weight between tissue samples was significantly different ( $p=0.001$ ), there was no difference in final concentration of DNA/g of sample $(p=0.895)$. DNA extraction from lung tissue samples (three samples per patient) was randomised (each extraction group never contained only one sample type or all samples from the same patient) to randomise the manipulation effect.

Since the lung samples are considered as low biomass samples, background controls were made throughout the sampling and extraction procedure. Negative sampling control was collected for each BAL and consisted of physiological serum collected with syringe used for instillation from the same liquid recipient. During the DNA extraction, milliQ water was used as a negative background control and underwent the same procedure as the real samples. All controls were sequenced and analysed. Reagents used in DNA extraction and sample pre-treatments were either autoclaved, filtered through $20 \mu \mathrm{m}$ filters or purchased sterile. All tools and pipettes were thoroughly washed and disinfected before and after each extraction cycle or between different extraction steps.

\section{6 ribosomal RNA (16S rRNA) gene sequencing}

DNAVision (Belgium), using Illumina MiSeq technology, performed $16 \mathrm{~S}$ ribosomal rRNA gene sequencing. Following the PCR amplification of the targeted region V3-V4, libraries were indexed using the NEXTERA XT Index kit V2. The sequencing was carried out in pairedend sequencing $(2 \times 250 \mathrm{bp})$ by targeting an average of 10,000 reads per sample. Next, sample sequences were clustered into OTUs based on $97 \%$ sequence similarity. This was performed with software QIIME (Quantitative Insights Into Microbial Ecology). Further microbiota analyses were done on generated "raw" OTU (outer taxonomic unit) table, taxonomy and Newick formatted phylogenetic tree provided by DNAVision.

\section{Sequence processing and microbiota analysis}

Microbiota analysis and visualisation were done with RStudio 3.5.2 [35]. (packages "phyloseq" [36], "vegan" [37], "microbiome" [38], "ggplot2" [39], "DESeq2" [40],"metacoder" [41]).

Raw OTU table was filtered to keep only kingdom $\mathrm{Bac}$ teria for further analysis. The total of 26 negative controls (sampling and background) had in average 21 detected OTU with average of 4 reads/OTU and did not belong to more abundant OTUs in samples. These OTU counts were subtracted from corresponding samples, i.e. negative sampling control (physiological serum for washing) from corresponding BAL sample, and negative extraction controls from the samples in the extraction group. Samples were processed in two batches with controlled randomisation of all sample types and patients, including clinical data, so that both batches were equally diverse. Therefore, only OTUs present in all samples of one batch and not present in all samples of the other were excluded from further processing as a consequence of unequal extraction efficiency (and not of contamination). These preprocessings did not alter any of the measures, and have left the data virtually unchanged. Next, OTU present in at least $10 \%$ of the lung samples and $20 \%$ of saliva samples or having more than 50 overall counts (for each group) were kept for further processing (in our case, this was equivalent of keeping the OTUs with minimal average abundance of $0.001 \%$ in either of groups). Only samples with more than 1000 reads were included in analysis. This excluded 6 samples (without preference for certain factor): 2 BAL, 3 peritumoural tissues (LUNG.PT) and 1 tumour (LUNG.T). Average read number of final sample groups was 39,083 \pm 9697 (mean \pm SD) for saliva, 17,046 $\pm 14,879$ for BAL, 13,352 $\pm 12,909$ for LUNG.DP, $13,039 \pm 11,394$ for LUNG.PT and $5846 \pm 4505$ for LUNG.T.

For analysis of alpha and beta diversity, samples were rarefied at 1195 reads with 100 iterations. Observed OTU number, Shannon diversity index and Faith's phylogenetic diversity were used for alpha diversity characterisation ("phyloseq"). Groups' beta diversity was calculated based on weighted (importance of abundance and quality) (wUF) and unweighted (importance of absence or presence of OTUs) (uwUF) UniFrac distances using function adonis ("vegan") with 999 permutations and presented with non-metric multidimensional scaling (NMDS). Core microbiota clustering was based on Bray-Curtis distance and NMDS method.

Taxonomic trees ("metacoder") representing relative abundance of taxa within each sample group were based on arithmetic mean of relative abundance calculated from unrarefied OTU table for each group. Input for trees representing differential abundance was calculated by DESeq function using zero-inflated method of negative binomial general linear model (significant coefficient difference calculated by Wald's test) and BenjaminiHochberg (BH) correction for multiple comparison with 0.05 threshold ("DESeq2"). The model was used on unrarefied taxa counts.

Difference in alpha diversity and paired UF distances was calculated by Kruskal-Wallis or Man-Whitney U test with $\mathrm{BH}$ correction for multiple comparison with 0.05 threshold of significance.

\section{Managing missing data - paired/unpaired tests}

A total of six lung samples was excluded from the study due to insufficient number of reads $(<1000)$. Excluded samples did not originate from only one specific tissue, only one patient or exclusively belonged to one criterion (only one sex, lobe location, or tumour type). Therefore, preservation of strictly paired analysis would exclude an important number of other related samples. For this reason, analysis was unpaired if not specified otherwise. 


\section{Results}

\section{Participant characteristics}

The total of 18 patients eligible to surgical treatment without neoadjuvant therapy was included in the study. Microbiota was analysed in 17 saliva and 68 lung samples, where 16 patients provided all 5 different samples (1 saliva plus 4 lung). For the analysis relative to the location of the tumour lobe, patients were grouped into two groups: 1st group with tumour in upper lobes (UL), and 2nd group with tumour in middle and lower lobes (LL) (share same descending bronchus). Patients with LL tumour had significantly lower predicted diffusing capacity of the lung for carbon monoxide (DLCO) than patients with tumours in UL $(p=0.034)$. Other clinical parameters showed no significant difference. Patients' characteristics (total and per lobe location group) and final sample number used in analysis after exclusion of samples with less than 1000 reads are shown in Table 1.

\section{Beta diversity identifies BAL as a unique sample}

Microbiota was analysed in saliva, bronchoalveolar lavage fluid (BAL), non-malignant Distal Piece (LUNG.DP), Peritumoural Tissue (LUNG.PT) and Tumour (LUNG.T). As expected, saliva showed a clear separation from the four lung samples (BAL and tissues) with significant difference in beta diversity based on both weighted and unweighted UniFrac distances (Fig. 1a). Moreover, the three lung tissues were all significantly different from BAL (Fig. 1a), but with peritumoural tissue showing the least significant dissimilarity (wUF). There was, however, no significant difference between tissues. Looking at samples' position in NMDS (Fig. 1a), it was visible that BAL creates a distinct cluster with other three lung tissues when compared to salivary microbiota. However, within this "lung" cluster, BAL samples were concentrated on the front towards saliva cluster.

The four lung samples shared similar average values of observed OTU number $(\sim 120)$ as well as phylogenetic $(\sim 15)$ and Shannon diversity indexes $(\sim 3.5)$ (Fig. 1b-d). Although, compared to saliva, lung microbiota had higher variance, all lung samples showed significantly higher phylogenetic diversity and higher number of observed OTUs (latter significant only for tumour) compared to salivary microbiota. However, there was no difference in Shannon diversity between saliva and lung samples, with averaging index value of $\sim 3.5$ (Fig. 1d).

\section{Proteobacteria and Firmicutes (class Clostridia) dominate lung samples}

Thirteen phyla, 29 classes (27 in BAL), 87 families (85 in BAL and tumour), and between 112 and 115 genera were detected in each of the lung samples. In saliva these numbers were lower, with 10 phyla, 17 classes, 26

Table 1 Characteristics of patients included in the study

\begin{tabular}{|c|c|c|c|c|}
\hline & Total & Upper lobe T & Lower lobe T & $p$ \\
\hline No. of patients & 18 & 10 & 8 & \\
\hline Male/female & $13 / 5$ & $8 / 2$ & $5 / 3$ & \\
\hline Age (years) & $68 \pm 8$ & $65 \pm 9$ & $72 \pm 6$ & 0.061 \\
\hline BMl & $25 \pm 3$ & $25 \pm 4$ & $25 \pm 3$ & 0.859 \\
\hline $\mathrm{T}$ in upper/middle/lower lobe & $10 / 2 / 6$ & 10/0/0 & $0 / 2 / 6$ & \\
\hline ADC/SCC/carcinoid & $11 / 5 / 2$ & $6 / 2 / 2$ & $5 / 3 / 0$ & \\
\hline Stage $|/||/|||$ & $8 / 2 / 8$ & $5 / 2 / 3$ & $3 / 0 / 5$ & \\
\hline Tumour size $(\mathrm{cm})$ & $3.7 \pm 2.3$ & $3.6 \pm 2.4$ & $3.9 \pm 2.4$ & 1 \\
\hline Smoker/ex-smoker/never-smoker & $2 / 14 / 2$ & $0 / 9 / 1$ & $2 / 5 / 1$ & \\
\hline Pack-year (smokers, ex-smokers) & $31 \pm 20$ & $31 \pm 21$ & $33 \pm 20$ & 0.823 \\
\hline $\mathrm{FEV}_{1}$ (\% of expected value) & $98 \pm 11$ & $95 \pm 8$ & $101 \pm 14$ & 0.408 \\
\hline DLCO (\% of expected value) & $74 \pm 16$ & $81 \pm 13$ & $64 \pm 16$ & 0.034 \\
\hline $\mathrm{FEV}_{1} / \mathrm{FVC}$ (\% of expected value) & $96 \pm 10$ & $95 \pm 11$ & $98 \pm 10$ & 0.630 \\
\hline \multicolumn{5}{|l|}{ Final no. of analysed samples } \\
\hline Saliva & 17 & 10 & 7 & \\
\hline BAL & 15 & 8 & 7 & \\
\hline LUNG.DP & 17 & 10 & 7 & \\
\hline LUNG.PT & 14 & 9 & 5 & \\
\hline LUNG.T & 16 & 9 & 7 & \\
\hline
\end{tabular}

$A D C$ adenocarcinoma, $B A L$ bronchoalveolar lavage fluid, $B M I$ body mass index, $D L C O$ diffusing capacity of the lung for carbon monoxide, $F E V_{1}$ forced expiratory volume per second, FVC forced vital capacity, LUNG.DP non-malignant tissue, LUNG.PT peritumoural tissue, LUNG. $T$ tumour, SCC squamous cell carcinoma, $T$ tumour 


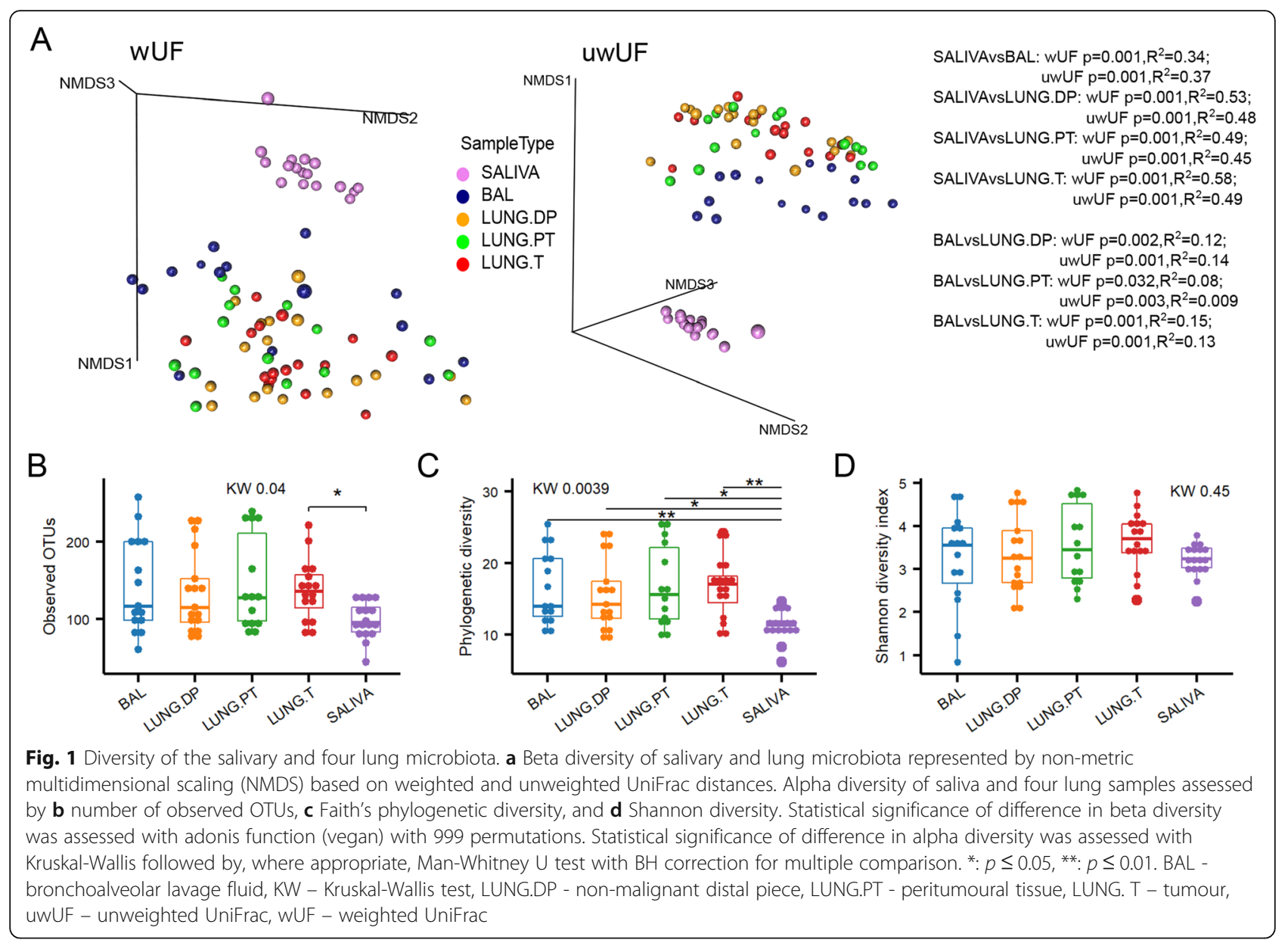

orders, 49 families and 68 genera. Composition of each sample is shown in Fig. 2a in a form of a taxonomic tree with indicated average relative abundance of taxa next to taxon's name $(>0.001 \%)$ and the number of samples in which they were detected (numbers within branches). The most abundant phyla and genera in each sample are synthetically presented in Fig. $2 \mathrm{~b}$ and c, respectively. The saliva tree was the least complex from all the trees, heavily dominated by the phylum Firmicutes (53.7\%) and belonging to genus Streptococcus (32.7\%) (Fig. 2a, Fig. 2b). Other phyla, as Bacteroidetes, Actinobacteria, Proteobacteria and Fusobacteria, were less abundant and are noted in the decreasing order (Fig. 2b). Except Streptococcus, additional 11 genera had abundance higher than $1 \%$, including Prevotella, Veillonella, Neisseria, Porphyromonas, and Actinomyces as the top five.

In the lung samples, two most abundant phyla were Proteobacteria and Firmicutes, but the dominating one changed relative to the sample. So, phylum Firmicutes was the most abundant in BAL, while Proteobacteria dominated non-malignant tissue and tumour (Fig. 2a, b). Interestingly, in peritumoural tissue two phyla were equally abundant. Phyla Bacteroidetes and Actinobacteria were found in lower abundance in all lung samples ( 10\% each). Interestingly, while high abundance of phylum Firmicutes in saliva was almost entirely due to members of the class Bacilli, in lung samples it is due to class Clostridia, introducing one of the fundamental differences between these two microbiota. Moreover, Clostridia was the most abundant class in all lung samples except the tumour, where it shared the highest abundance with the class Alphaproteobacteria. Compared to saliva, the whole phylum Proteobacteria was more developed in lung samples, containing additional large class of Alphaproteobacteria, but lacking Epsilonproteobacteria (detected in saliva).

On the genus level, there was no extensive prevalence by one genus as seen in saliva, but rather a group of representatives with different taxonomic origin (Fig. 2a, c). In the three lung tissues, Pseudomonas, Clostridium, Kocuria, Acinetobacter and Sphingomonas were the five most abundant genera, but in BAL, those were Pseudomonas, Blautia, Streptococcus, Capnocytophaga and Acinetobacter (Fig. 2c). Interestingly, two highly abundant genera in tissues, Clostridium $(\sim 15 \%)$ and Kocuria $(\sim 5 \%)$ were found in very low abundance in BAL. Inverse was seen for Capnocytophaga, that seemed to be 





a BAL-related genus $(\sim 5 \%)$, with a very low presence in saliva and absence in tissue samples. Furthermore, BAL was the only lung sample that had higher abundance of so-called supraglottic taxa, as Streptococcus, Prevotella and Veillonella, compared to other tissue samples. The abundance was also slightly higher in peritumoural tissue, supporting potentially increased similarity between BAL and peritumoural tissue seen in beta diversity (Fig. 1a).

\section{Whole phyla and classes significantly different between lung samples and saliva, but also between BAL and tissues}

Figure 3a shows difference in taxa abundance between samples. Taxa with significant difference are coloured, with a colour scale representing $\log 2$ fold change in abundance between compared sample pair.

The first row (Fig. 3a) shows taxa with significantly different abundance between saliva and each of the four lung samples. Compared to saliva, all lung samples had significantly higher abundance in whole classes of Alphaproteobacteria, Deltaproteobacteria, Cytophagia, Sphingobacteriia, [Saprospirae], and Acidimicrobiia, but also in whole phyla such as Cyanobacteria, Acidobacteria, Nitrospirae, Verrucomicrobia and Planctomycetes (latter not seen for BAL). Similarly, phyla Synergistetes, Spirochaetes, Fusobacteria and TM7 (not in BAL), classes Epsilonproteobacteria and Erysipelotrichi were significantly more abundant in saliva than in any of lung samples. On the other hand, multiple descending members of several higher taxa were not strictly more present in only one sample type. This particularly concerned members in the classes from the principal phyla Proteobacteria, Firmicutes, Bacteroidetes and Actinobacteria. Significantly more abundant in saliva samples were orders Neisseriales (Betaproteobacteria), Pasteurellales (Gammaproteobacteria, contains Haemophilus), Lactobacillales (Bacilli, contains Streptococcus) and families Veillonellaceae (Clostridia), Flavobacteriaceae (Flavobacteriia), Prevotellaceae, Porphyromonadaceae (both Bacteroidia), Actinomycetaceae and Corynebacteriaceae (both Actinobacteria). Conversely, from the same higher taxa, significantly more abundant in lung samples were orders Burkholderiales (Betaproteobacteria), Pseudomonadales, Legionellales, Xanthomonadales, Enterobacteriales (both Gammaproteobacteria), Bacillales, Turicibacteraceae (both Bacilli), and families Ruminococcaceae, Lachnospiraceae (both Clostridia), Bacteroidaceae (Bacteroidia), Propionibacteriaceae, Dietziaceae and Bogorellaceae (Actinobacteria).

Second row in Fig. 3a shows significant difference in abundance between BAL and the three lung tissues. With a few pair-reserved exceptions, pattern was highly similar between comparisons. Also, it was visible that most of the differences indicated significantly increased abundance in BAL compared to tissues. In BAL, significantly higher abundance was seen in phylum Fusobacteria, classes Clostridia and Bacilli (genera Streptococcus, Veillonella, Roseburia, Oribacterium, Phascolarctobacterium, Parvimonas, and Megasphera), orders Pasteurellales (Haemophilus) and Desulfovibrionales, families Actynomicetaceae, Flavobacteriaceae (Capnocytophaga), and genera Atopobium, Porphyromonas, Neisseria, and Rothia. Adversely, the three tissue microbiota had only a few taxa with significantly higher abundance compared to BAL. Those were the whole phylum Acidobacteria, families Acetobacteraceae and Clostridiaceae (genus Clostridium), and genus Perlucidibaca.

However, there were differences in taxa that could be observed only between certain BAL-tissue pairs. Interestingly, the highest number of individual differences was seen in comparison between BAL and tumour microbiota. Here, only genus Coprococcus was significantly more abundant in BAL, while genus Kocuria, orders Bdellovibrionales, Myxococcales, Rickettsiales, and class [Saprospirae] were all significantly more abundant in tumour. Considering non-malignant tissue microbiota, only family Dietziaceae was more abundant, while orders Bifidobacteriales and Erysipelotrichales were significantly more abundant in BAL. Interestingly, tumour and non-malignant tissue had important number of similar differences in comparison to BAL. While BAL had higher abundance of genera Blautia, Granulicatella, Ruminococcus, Oscillospira, Prevotella, and Mezorhizobium, more abundant in both tumour and nonmalignant tissue were phylum Cyanobacteria and family [Weeksellaceae]. On the contrary, peritumoural tissue did not share any of these differences with BAL as did the other two tissues. In individual differences, Kocuria was the only significantly more abundant genus in peritumoural tissue, and inversely, only Staphylococcus was more abundant in BAL.

There were, however, no significant differences between three tissues.

\section{Core OTUs in lung samples mostly members of phylum Proteobacteria}

Core microbiota was determined as OTUs detected in $100 \%$ of samples in each group (Fig. 3b). The highest number of core OTUs was observed in saliva, with the total of 36. Two-fold less was seen in non-malignant tissue (16), peritumoural tissue (14) and tumour (14), and four-fold less in BAL (9). $75 \%$ of core OTUs in saliva belonged to phylum Firmicutes, with as high as 17/20 OTUs from genus Streptococcus, while additional 15\% was from the phylum Actinobacteria (especially genus Actinomyces). In lung samples, $70 \%$ of the core OTUs belonged to the phylum Proteobacteria (1/3 from class Alphaproteobacteria) and other $30 \%$ to Firmicutes. Core 


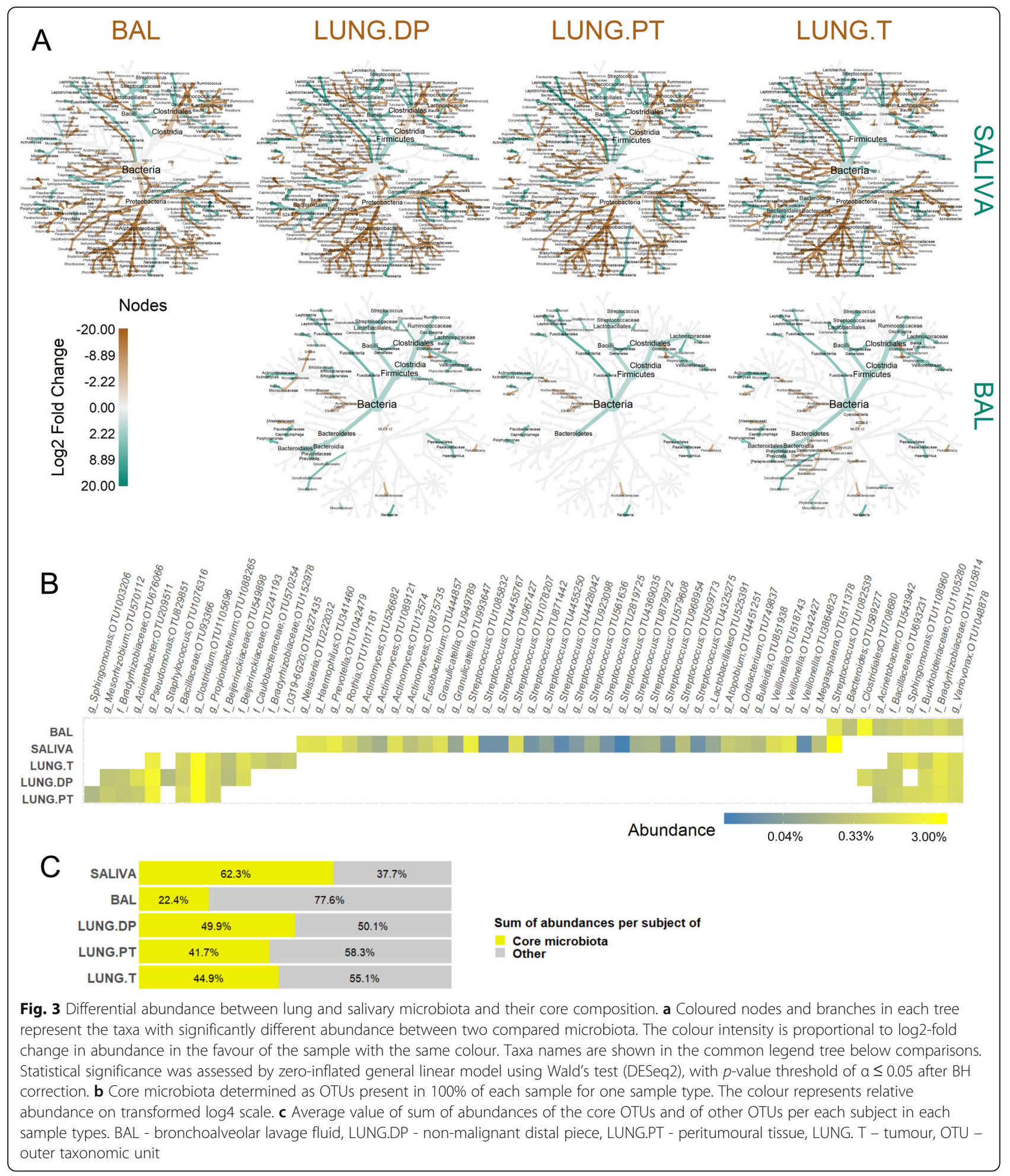

OTUs were mostly shared between different lung sample types, especially between tissues. OTUs corresponding to genus Variovorax and unclassified members of families Bradyrhyzobiaceae, Burkholderiaceae, and Bacillaceae were detected in all four lung microbiota, while
OTUs for genera Pseudomonas, Clostridium and Propionibacterium were only common in all lung tissue microbiota. Even though $30 \%$ of lung core OTUs belonged to Firmicutes, only one OTU corresponded to genus Streptococcus and was a part of BAL core 
microbiota. This was also the only core OTU shared between saliva and lung samples, i.e. only BAL, which were otherwise clearly distinct. Within core microbiota (Fig. $3 \mathrm{~b})$, certain OTUs were uniquely associated with species, such as OTUs for Rothia mucilaginosa, Propionibacterium acnes, Staphylococcus epidermidis, Prevotella melaninogenica, Variovorax paradoxus, Veillonella parvula (OTU 518743) and Veillonella dispar (other two OTUs). On average (Fig. 3c), core microbiota represented $62 \%$ of relative abundance in saliva, against only $22 \%$ seen in BAL. In the tissues, the core microbiota represented around half of the total abundance, ranging from 42 to $50 \%$ of abundance.

\section{Microbiota in lower lobes with higher abundance of Firmicutes and the diversity of peritumoural tissue as the most influenced by location}

Next, we examined whether there is a significant difference between lung microbiota associated to the tumour lobe relative to its location. Interestingly, only peritumoural tissue microbiota showed significantly different beta diversity between two locations in both wUF and uwUF (Fig. 4a, b). Moreover, difference in beta diversity between UL peritumoural tissue and BAL was not significant, unlike the one in LL (in UL: wUF $p=0.17$, uwUF $p=0.073$, vs. in LL: wUF $p=0.004$, uwUF $p=$ 0.002). To confirm this observation, we selected exclusively distances between two samples originating from the same patient (Fig. 4d, e), i.e. paired distances. Indeed, in UL peritumoural microbiota was significantly more similar to both saliva and BAL, manifested as shorter distances compared to ones in LL (Fig. 4d, e: "distance to SALIVA", "distance to BAL"). We next looked at the paired distances between the three tissue samples (Fig. 4d, e: "distance to LUNG.DP"). The paired distance between non-malignant tissue and the other two tissues, respectively, was inverse depending on the lobe location. In UL, there was an increased similarity between nonmalignant tissue and tumour, and in LL, between nonmalignant and peritumoural tissue. However, paired distance between peritumoural tissue and tumour remained unchanged (Fig. 4d, e: "distance to LUNG.PT"), suggesting a potentially balanced change or exchange of the microbiota maintaining the distance (and the difference) on the same level. In beta diversity however, these two samples were significantly different only in UL (Fig. 4c), while in LL difference was not significant $(p=0.077$, $\left.R^{2}=0.173\right)$. Altogether, this indicated that in upper lobes peritumoural tissue microbiota was significantly more similar to BAL microbiota, while in lower lobes peritumoural tissue was more similar to tumour microbiota.

Both BAL and peritumoural tissue varied in alpha diversity depending on lobe location, unlike non-malignant tissue and tumour. BAL in LL had significantly lower phylogenetic diversity and number of observed OTUs (Fig. 4f, g) compared to UL BAL, and a tendency seen for Shannon diversity (Fig. 4h). Inversely, LL peritumoural tissue had significantly increased Shannon diversity (Fig. 4h), with tendency in phylogenetic diversity and number of OTUs.

In LL, there was a marked decrease in abundance of Proteobacteria and increase in phylum Firmicutes in each of the lung samples (Fig. 4i). Therefore, LL tumour and non-malignant tissue had equal abundances in Proteobacteria and Firmicutes ( 35\%), while BAL and peritumoural tissue were both dominated by Firmicutes (56 and 45\%, respectively).

\section{Actinobacteria and Flavobacteriia show inverse abundance between BAL and extratumoural tissues depending on the lobe location, while tumour microbiota remains unchanged}

Tumour location in UL or LL significantly influenced the microbial abundance in each of the analysed sample types, but not in the same manner (Fig. 5). As suggested by diversity results, microbiota of the peritumoural tissue seemed to be the most influenced by the lobe location. The changes were limited to members of the three major phyla: Firmicutes, Actinobacteria and Bacteroidetes, and candidate phylum TM7. More precisely, in UL peritumoural tissue more abundant were members of the phylum Firmicutes, phylum TM7, classes Bacteroidia (phylum Bacteroidetes) and two families from phylum Actinobacteria (Actinomycetaceae and Bifidobacteriaceae). This abundance pattern was very similar to pattern of initial comparison between BAL and each of the lung tissue samples (Fig. 2a). This, added to the results of beta diversity, could confirm that microbiota in UL is more similar between peritumoural tissue and BAL then it is in the lower lobes. In the LL, peritumoural tissue microbiota was enriched with three classes from phylum Bacteroidetes (Flavobacteriia, Sphingobacteriia and Cytophagia), and with families Clostridiaceae (genus Clostridium) and Micrococcaceae (genus Kocuria) from phylum Firmicutes and Actinobacteria, respectively.

Compared to number of affected taxa in peritumoural tissue, both BAL and non-malignant tissue were less influenced by the lobe location, while tumour seemed to be almost entirely unaffected (Fig. 5). Moreover, phylum Firmicutes and class Bacteroidia, which in peritumoural tissue had the highest number of members with significant differences, were not found significantly different in either non-malignant tissue or BAL. Instead, UL non-malignant tissue was enriched with the phylum Fusobacteria and only a few other lower taxa without involvement of the whole clades (order Gemellales, families Actinomycetaceae and Pseudomonadaceae). 


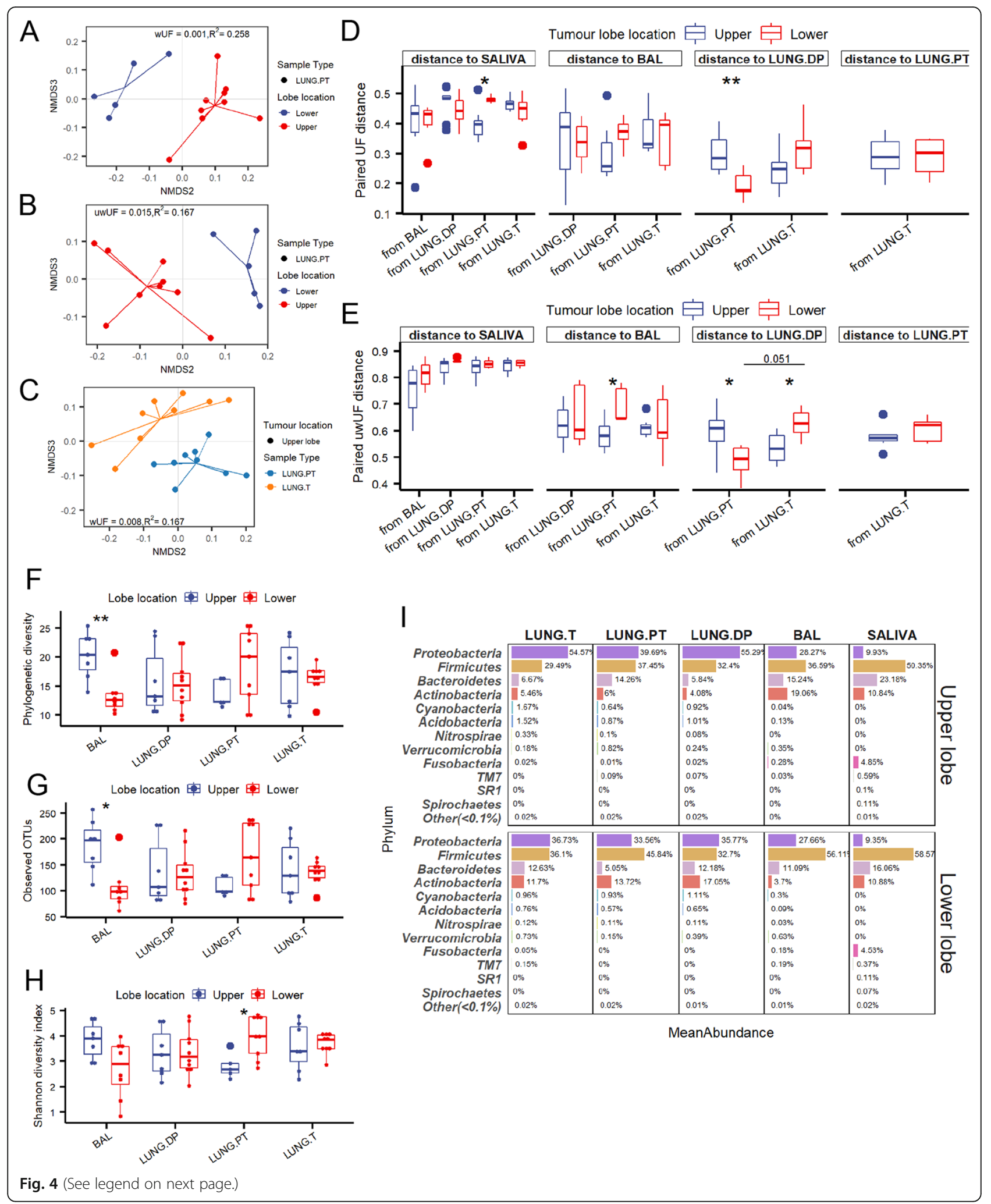




\section{(See figure on previous page.)}

Fig. 4 Diversity and predominant taxa in lung samples from upper and lower tumour lobes. Beta diversity found significantly different between peritumoural tissue from upper and lower lobes based on both a weighted (WUF) and b unweighted (UwUF) UniFrac distances. c Significantly different beta diversity based on wUF between peritumoural tissue and tumour in the upper lobe. $\mathbf{d}$ Weighted and e unweighted UF distances between samples coming from the same patient (i.e. paired distances) compared between upper and lower tumour lobes. The facet name represents the referent sample (e.g. "distance to BAL") to which were calculated the distances noted on x-axis (e.g. "from LUNG.T"). Smaller distance indicates increased similarity. Alpha diversity for four lung samples between upper and lower tumour lobe assessed by $\mathbf{f}$ Faith's phylogenetic diversity, $\mathbf{g}$ number of observed OTUs, and $\mathbf{h}$ Shannon diversity. $\mathbf{i}$ Most abundant phyla in each of the microbiota samples if the tumour is found in upper or lower lobes. Significance of difference in beta diversity was assessed with adonis function (vegan, 999 permutations). Statistical significance in alpha diversity and paired distances was assessed with Kruskal-Wallis followed by, where appropriate, Man-Whitney $U$ test with BH correction for multiple comparison. *: $p \leq 0.05,{ }^{* *}: p \leq 0.01$. BAL - bronchoalveolar lavage fluid, LUNG.DP - non-malignant distal piece, LUNG.PT - peritumoural tissue, LUNG. T - tumour

Interestingly, in both non-malignant and peritumoural tissue, phylum Actinobacteria (with genus Kocuria) and class Flavobacteriia (with genus Chryseobacterium) were significantly more abundant in LL compared to UL. However, opposite from the two tissues, in BAL these same two taxonomic groups were significantly more abundant in UL (Fig. 5). Instead of having a common abundance value in one lobe and a lower or higher value in the other lobe, the abundances between BAL and tissue samples were inverse in each lobe. E.g. the abundance of clade Actinobacteria in BAL and tissues was $19 \%$ versus 5\% in upper lobes, and $4 \%$ versus $\sim 13 \%$ in lower lobes, respectively). Except the mentioned two clades of Actinobacteria and Flavobacteriia, no other larger taxonomic groups were found as more abundant in UL BAL. Similarly, taxa found as more abundant in the lower lobes were dispersed between different phyla (Cyanobacteria, members of Proteobacteria, Firmicutes, and Actinobacteria).

Even though importance was given to $\log 2$ fold change of abundance, certain taxa held however high impact on the overall composition of the samples due to their higher relative abundance (Fig. 5, Additional file: Figure $1)$. This was particularly true for three genera, Clostridium, Kocuria and Pseudomonas. In peritumoural tissue, genus Clostridium (phylum Firmicutes) ranged from 3\% in UL vs $38 \%$ in LL, in non-malignant tissue genus Pseudomonas (Gammaproteobacteria) represented 18\% in UL vs $2 \%$ in LL lobes, and genus Kocuria (phylum Actinobacteria) ranged from 0.5 and $0.2 \%$ in UL to 12 and $15 \%$ in LL in peritumoural and non-malignant tissue, respectively.

Finally, saliva samples, as the extrapulmonary sample with no direct physical connection to the tumour location as lung microbiota, also showed significantly different abundance profile relative to tumour lobe location. If tumours were found in LL, saliva was significantly enriched in class Bacilli and families Enterobacteriaceae, Moraxellaceae (both Gammaproteobacteria) and Propionibacteriaceae (Actinobacteria). Curiously, no taxa were detected as significantly more abundant if tumour was found in UL.

\section{Stratification between lobes defines differences between BAL and tissues and confirms similarity of BAL and peritumoural tissue in upper lobes}

Stratification of samples by lobe location also revealed or defined differences between samples (Fig. 6). Existence or disappearance of certain differences was a direct result of individual lobe-related characteristics reported in the Fig. 5. Between saliva and the four lung samples, there was little change (Fig. 6), since these samples were initially already very different (Fig. 3). Several taxa were no more significant between saliva and BAL from upper lobes, while they were significant in all other comparisons (e.g. Nitrospirae, Neisseriales, Haemophilus). Comparing saliva and the three lung tissues, only taxa Spirochaetes, Sphingobacteria and TM7 showed changes relative to the lobe location. Among them, class Sphingobacteria was significantly more abundant in all lung tissues when compared to saliva, except in UL peritumoural tissue. This was interesting because neither UL nor LL BAL showed this difference, adding to similarity of BAL and peritumoural tissue in UL. Despite these changes and stratification by location, saliva was still predominantly abundant in Firmicutes, Fusobacteria, Epsilonproteobacteria, Erysipelotrichi, Prevotella and Neisseria clade, while lung samples dominated in abundance of Proteobacteria, Acidobacteria, Nitrospirae, Verrucomicrobia, Cyanobacteria, and the rest of Bacteroidetes (except Prevotella clade).

Unlike the comparison between saliva and lung samples, the aspect of lobe location induced much more developed and varying differences between BAL and tissues. Similarly to comparison with saliva, differential abundance pattern varied for both Nitrospirae and Cyanobacteria between BAL and each of the three tissues relative to lobe location. Further, as expected, Flavobacteriia and Actinobacteria showed inverse abundance between the two, being both more abundant in UL BAL and in LL tissues (Figs. 5 and 6). Interestingly, differences in phyla TM7 and Acidobacteria came in pairs. The significantly increased abundance of TM7 in BAL was accompanied with increased abundances of Acidobacteria in the tissues, otherwise no difference was seen in either of them. In UL, this was seen in 


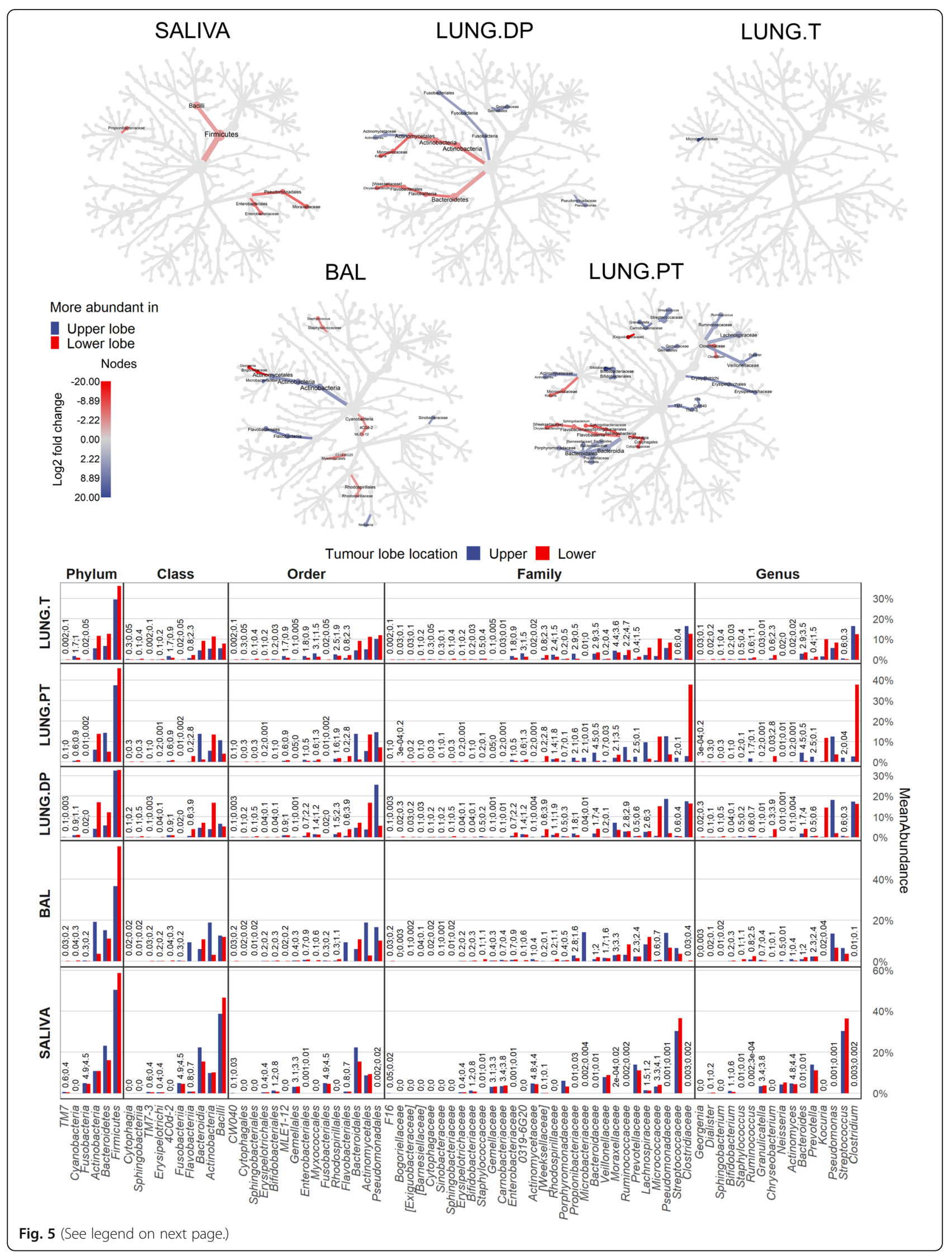


(See figure on previous page.)

Fig. 5 Differential abundance between upper and lower tumour lobes in salivary and lung microbiota. Each tree represents taxa with significantly different abundance relative to sample's origin (for lung) in the upper or lower tumour lobe. For saliva, the comparison shows the significant difference in salivary microbiota between patients with tumour either in upper or lower lobe. Coloured nodes and branches represent the taxa with significantly different abundance and the intensity is proportional to log2-fold change in abundance in the favour of the lobe noted with the same colour. Statistical significance was assessed by zero-inflated general linear model using Wald's test (DESeq), with p-value threshold of $a \leq 0.05$ after BH correction. Bar chart shows the relative abundance of taxa noted in the taxonomical trees. BAL - bronchoalveolar lavage fluid, LUNG.DP - non-malignant distal piece, LUNG.PT - peritumoural tissue, LUNG. T - tumour

tumour, while in LL this was seen for peritumoural and non-malignant tissue.

Interestingly, the pattern of differential abundance was much more similar between different comparisons of BAL and tissues in the lower lobes than in the upper lobes. Here, the highest number of differences was found between tumour and BAL, and unlike in other tissues, included a large portion of phylum Proteobacteria, especially class Alphaproteobacteria, as more abundant in tumour. Also, peritumoural tissue involvement of members from the phylum Firmicutes was minimal compared to other two tissues, excluding an important differentiating factor. This could be, therefore, one of the major reasons behind increased similarity between BAL and peritumoural tissue in UL. Detailed individual differences between BAL and tissues can be found in Additional file 1: Text 1 "Additional explanation of differences between lung samples".

Finally, significant differences were noted between tissues (Fig. 6), but they were, however, few in number. They were dispersed within four most abundant phyla and considered mostly endpoint taxa. Also, there were less differences in LL than in UL. Curiously, in both locations, more difference was found between tumour and peritumoural tissue, than between tumour and nonmalignant tissue (none and one in UL and LL, respectively). Looking at the three tissues, in upper lobes class Cytophagia, family Exiguobacteraceae and Clostridiaceae were the least abundant in peritumoural tissue, while in LL family Microbacteriaceae had significantly the lowest abundance in tumour. Detailed individual differences between the tissues can be found in Additional file 1: Text 1 "Additional explanation of differences between lung samples".

\section{Discussion}

Following the last advancement on the interaction between gut microbiota, immune system and the tumour environment [8-11], there has been a growing interest in studying this concept in other physiological environments involving extraintestinal tumours. However, before exploring the effect of the gut microbiota, there has been an increasing necessity to investigate the effect of the local microbiota on the tumour as well [42]. Despite the emerging number of studies on the lung microbiota in different malignancies [17, 43-46], its involvement in lung cancer is in its promising beginnings [26, 30, 32, $33,47]$. However, for the moment there is still no study considering the ground difference between different lung samples and their microbiota, while it is suggested that those could harbour microbiota with varying characteristics [21], and therefore, have diverging interactions with local immunity and tumour.

This study is, to our knowledge, the first to characterise the lung microbiota originating from four different lung samples (BAL, non-malignant tissue, peritumoural tissue and tumour), accompanied with the characterisation of salivary microbiota in NSCLC patients. We hypothesised that samples' nature, "architecture", physiological functions and environment, will influence characteristics of associated lung microbiota. Therefore, BAL should represent "planktonic" bacterial population found within the bronchial lumen or associated with biofilms or mucus [48], sampled along with the hydrodynamic force of the instilled liquid. Non-malignant tissue from the same lobe but taken on the opposite side from the tumour should represent a sample with a normal lung architecture, with well-defined small alveolar spaces and single-cell epithelial layer. In majority, it should harbour the biofilm-, mucus- and cell-associated lung microbiota. On the contrary, tumour represents a tissue with disrupted architecture, varying in form and obstruction degree relative to its type and grade [2]. Tissue modelling could also involve overproduction of the mucus as seen in certain subtypes of adenocarcinoma [49], but also different reaction of the immune system [50]. Finally, peritumoural tissue represents a nonmalignant tissue in the direct contact with the tumour, separated as based on different histological properties. In literature it is addressed as tumour microenvironment and harbours different roles in stimulation or suppression of tumour metabolism [51]. Therefore, we presumed that its characteristics would be different both from the distal non-malignant tissue and the tumour. Except for the number of different lung samples, the particularity of this study is also the way of obtaining BAL. It was obtained directly on the excised lobe containing tumour without using the bronchoscope, to minimise contamination risk of upper airways and increase the precision in characterisation of "true" BAL microbiota in the tumour proximity. 


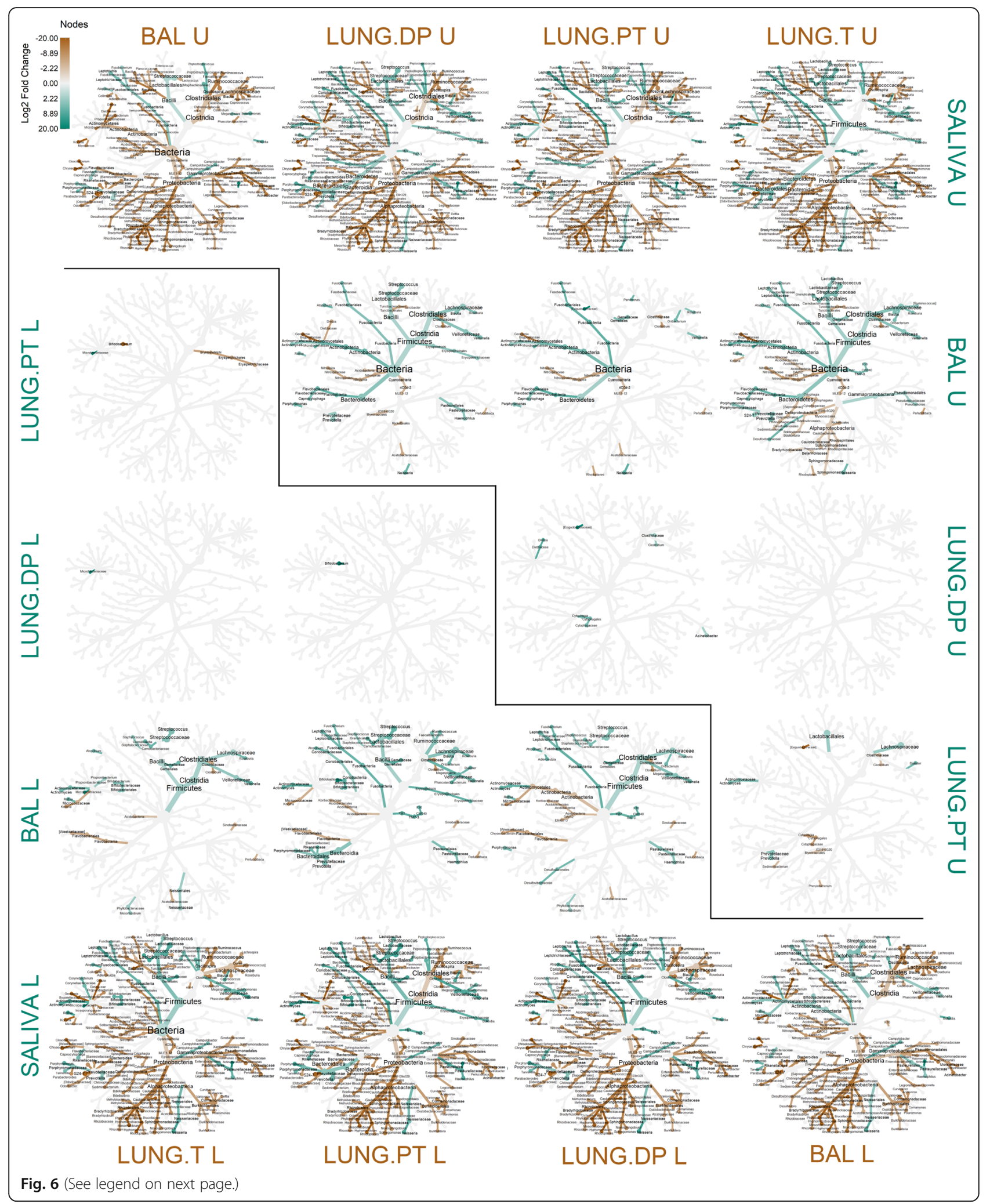


(See figure on previous page.)

Fig. 6 Comparison of abundance between salivary and lung microbiota relative to tumour lobe location. The two parts of the figure represent comparison in abundance between samples linked to upper $(U)$ lobes in the upper part of the figure and to lower $(L)$ lobes in the lower part of the figure. Coloured nodes and branches represent taxa with significantly different abundance between the two compared samples. The colour intensity is proportional to log2-fold change in abundance in the favour of the sample with the same colour. Statistical significance was assessed by zero-inflated general linear model using Wald's test (DESeq), with $p$-value threshold of a $\leq 0.05$ after BH correction. BAL - bronchoalveolar lavage fluid, L - lower lobe, LUNG.DP - non-malignant distal piece, LUNG.PT - peritumoural tissue, LUNG. T - tumour, U - upper lobe

We reported that the four lung samples significantly clustered versus oral microbiota based on beta diversity, a confirmation of a previous result in healthy subjects that lung microbiota is distinct from other communities [32]. Salivary microbiota had several lower alpha diversity metrics compared to lung microbiota, as well as high dominance of genus Streptococcus and overall phylum Firmicutes. Along with its homogenous core microbiota (again prevalence of Streptococcus OTUs) that represented almost $3 / 4$ of total relative abundance per patient, this indicated its inter-subject stability and lower complexity compared to the one of the lung samples. In addition, the significant difference in abundance including the majority of the taxa detected in samples clearly separated the four lung samples versus oral microbiota. We identified typically oral taxonomic groups more abundant in saliva in all comparisons, such as Fusobacteria, Spirochaetes, Synergistetes, Erysipelotrichi, Epsilonproteobacteria, Bacilli and Neisseriales, most of them being in concordance with previous literature [52, 53]. On the other hand, phyla Acidobacteria, Cyanobacteria, Nitrospira, Verrucomicrobia and Planctomycetes were detected as strictly lung-associated (detected in each lung sample) with no representatives in saliva.

Further, we showed that BAL microbiota, even though undoubtedly belonging to lung microbiota cluster, had significantly different features from tissues. First, multidimensional representation of the beta diversity placed BAL samples on the side of the lung cluster towards salivary microbiota (Fig. 1a). Even though presence of more abundant salivary taxa was very limited in both tissue samples and BAL, Streptococcus, Prevotella and Veillonella (the three most abundant genera in saliva) were found elevated in BAL compared to tissue samples. It was previously suggested that these and other typically oral bacteria are found in low abundance in healthy lungs, due to their constant elimination [54]. Since BAL represents microbiota of the bronchial lumen, which undergoes constant influx of upper airways particles by respiration and is also the first "space" influenced by microaspiration [55], those could explain increased presence of these supraglottic taxa in BAL compared to the lung tissues. This position "in the middle" was also seen when looking at the differential abundance between all five samples (Fig. 5). The taxa that were more abundant in saliva when compared to the four lung samples almost perfectly corresponded to taxa that were more abundant in BAL when compared to the three lung tissues. Second, lung samples had Proteobacteria and Firmicutes as the two most abundant phyla, however with different ratios. While BAL was dominated by Firmicutes, almost twice as abundant as Proteobacteria, lung tissues were dominated by Proteobacteria (as previously reported [32]), or at best, had equal abundance of the two (peritumoural tissue). Here it is however important to note that the high abundance of phylum Firmicutes in lung samples was due to members of the class Clostridia, unlike in saliva, where it was due to highly abundant class Bacilli. This emphasises one of the essential differences between oral and lung microbiota often omitted by selective presentation of only phylum or genus level. A third feature was that BAL did not differentiate from lung tissues only by difference in taxa abundance, but also by the presence of BAL-specific bacteria, such as genus Capnocytophaga, or absence of tissue specific-bacteria, such as genus Kocuria or Clostridium. The two latter genera were, however, detected in BAL, but with relative abundance 175 -fold and 520 -fold lower $(\sim 0.03 \%)$, respectively, than in the tissues. This detection is possibly due to their presence in host's cells during the DNA extraction, since cellular BAL was used for BAL analysis [48]. Lastly, no differences in alpha diversity metrics were detected between four lung samples, suggesting two different but equally "rich" lung microbiota populations. All this supports the hypothesis that BAL indeed represents a unique lung microbiota in lung cancer and that concerns of diverging results due to different samples have been justified [21].

Conversely to our hypothesis, there was no significant difference detected between the three lung tissues when analysing the totality of samples. Although increased diversity of non-malignant tissue versus tumour has been previously suggested [32], we did not find the same. This could be due to the fact that our study group was more balanced in patients with less and more advanced tumour stages. In a referenced study, the majority of subjects had tumours in stages I and II, showing decreased diversity compared to higher stages according to authors.

We also reported on the existence of the core OTUs and that these were mostly shared between lung tissues and partially with BAL, consisting in the majority of the 
members of Proteobacteria. Interestingly, one of the core OTUs was uniquely associated to species Variovorax paradoxus, an ambiguous aerobic organism found in many niches, also in oral microbiome, and known for its potent biofilm creation [56, 57]. It was detected as the core OTU in all four lung samples, while conversely, it was not found in saliva (where it was originally detected). This could suggest its potential role in biofilm creation in lower airways and would be an interesting topic for future verification and investigation of potential role in pathogenesis of lung cancer [58].

Even though still under debate, several studies connected lower lobe tumours with worse prognostics [5, 59, 60]. Therefore, we examined the characteristics of the lung, but also salivary microbiota, if the tumour was found in upper or lower lobes. Indeed, we noted several changes in alpha and beta diversity but they were focused on changes in BAL and peritumoural tissue (with lower and higher alpha diversity, respectively, in LL compared to UL). We reported that in all four lung samples, lower lobes had decreased abundance of phylum Proteobacteria into favour of Firmicutes. Increased abundance of phylum Firmicutes was previously seen in BAL of LC patients compared to patients with benign-mass lesions [47], but also in patients with COPD [21]. In our study, $62 \%$ of patients in the LL were diagnosed with stage III tumours, against $30 \%$ in the UL. Even though this remains a small study, this finding supports that "shift" from Proteobacteria to Firmicutes might have a role in lung cancer progression.

We showed that location significantly influenced the abundance of two clades, Actinobacteria and Flavobacteriia, in the inverse manner between BAL and extratumoural tissues. While both are more abundant in UL BAL, their abundance in UL extratumoural tissues was 210-fold lower, with exact inverse situation in LL. Both genus Chryseobacterium sp. (Flavobacteriia) and Kocuria rhizophila (Actinobacteria) detected as more abundant in LL tissues have been previously reported as uncommon human pathogens $[61,62]$. However, their increased presence selectively in LL tissues and their important overall abundance ( $\sim$ and $12 \%$, respectively) suggest that there might be an important communication between different lung environments depending on local conditions or malignancy status, influencing preferential bacterial growth in one type of considered environment.

Interestingly, we found significant differences in abundance of salivary microbiota between patients with tumour in either upper or lower lobe. All differences considered taxa elevated in the case of LL tumours and included families previously associated with bacterial exacerbations and infections (Moraxellaceae, members of Bacilli as Streptococcus, Staphylococcus, etc.) [63] or pulmonary complications (Propionibacteriaceae, Enterococcaceae) [64-66]. However, these taxa were not found elevated in LL lung microbiota. This, however, does not exclude their implication in malignant changes or being its consequence, and will be an interesting topic for further consideration.

Finally, we found that peritumoural tissue showed higher similarity to BAL in UL both in beta diversity and in abundance, while in LL it shared characteristics with other tissue samples. Moreover, we found that alpha diversity of peritumoural tissue increased in LL to the level of other tissues, while the ones of BAL decreased. Peritumoural tissue is the tissue in direct contact and interaction with the tumour. In the history of cancer research, the role of extracellular matrix (ECM) surrounding tumour has been extensively studied [67]. Tumour cells are found to be able to directly influence the rearranging of connective units (such as collagen) and degradation of ECM to create a tumour-permissive environment and enabling metastatic progression [68-70]. In our case, it is possible that the observed changes in peritumoural tissue composition reflect certain remodelling of ECM, which could therefore be either more or less permissive for microbial attachment.

Curiously, of all samples, tumour was almost completely uninfluenced by location. Rather than from the outside, it is possible that intratumoural conditions, such as oxygen availability, density, necrosis and other factors $[71,72]$ independent of the external conditions are more likely to be the influencing ones. This is, however, a matter for further research.

The major strongpoint of this study is the analysis of the microbiota from four different lung samples covering the major physiologically different environments in NSCLC patients that has not been characterised previously, with the addition of saliva as the sample of oral microbiota. The representation using taxonomical trees also gives better insight into sample's composition, especially important in characterisation of this type of as yet, scarcely defined microbiota. Moreover, this study reports higher numbers of detected OTUs as well as diversity indexes than previously suggested for saliva, non-malignant tissue, tumour and BAL [29, 32, 33]. The reason could be due to the higher quality of obtained samples (only 6 from 85 samples lower than 1000 reads) and direct sampling of the BAL in the excised lobe. Also, the observed study group and stratification by tumour lobe location were well balanced in various clinical and demographic factors, minimising the result's bias.

The major drawback of this study is a low number of subjects, due to strict inclusion criteria for the overall protocol and a limited recruiting time. Additional inconvenience is that, even though initially requested, forming a control group has not been authorised by the Ethics 
Committee due to the invasiveness of the sampling techniques. Certain studies on lung cancer microbiota have adapted different strategy to include a non-malignant control group in their protocols, e.g. prospective studies analysing BAL from patients with pulmonary nodules that are next characterised as either benign or malignant (i.e. lung cancer) [47]. However, patients diagnosed with benign pulmonary nodules represent the minority, in most cases they do not require any treatment and partial resection is undertaken only in rare occasions [73, 74]. With the objective to analyse the lung microbiota from multiple lung samples (BAL in situ and different tissues), the partial resection in benign lung nodules would exclude both the possibility of performing BAL directly on the excised lobe and obtaining different types of lung tissue relative to the distance from the nodule. Moreover, it is unclear for the moment whether the presence of benign pulmonary nodule also modifies the local lung microbiota, introducing a potential bias in interpretation without previous investigation. Considering other surgically treatable lung diseases, such as interstitial lung disease [75], collapsed lung [76], emphysema or bronchiectasis [77], they represent malignancies with a distinct physiological condition and were not considered for use as a potential control group. The ideal control group would be the one including lobectomy in "healthy" patients, as seen in certain transplantation cases [78].

The limiting factor was also the decision to obtain BAL directly from the excised lobe to improve the precision in characterisation, but disabling the possibility of sampling the complementary non-tumour lobe by bronchoscopy due to difference in sampling technique.

Finally, it is important to note that the surgical resection considers fewer than $20 \%$ of all lung cancer patients and includes less severe cases [79]. Therefore, our results cannot be extrapolated to the overall lung cancer community, especially the most advanced cases relying mostly on multimodality treatment [2].

\section{Conclusion}

To our knowledge, this is the first trial that studied oral and lung microbiota from both BAL and three different tissue samples in 18 NSCLC patients. We confirmed that oral and lung microbiota are significantly different both in diversity and in taxonomy. However, we showed that BAL indeed represents a unique microbiota compared to three different tissue microbiota (from non-malignant, peritumoural and tumour tissue). We found that location of the tumour in upper or lower lobes influenced both oral, BAL and extratumoural microbiota with detection of Firmicutes as dominating phylum, but surprisingly, not the one of the tumours. Moreover, few differences were found between tissues, suggesting that these are not conditioned by lobe location and turning attention to other factors for future consideration, such as tumour type, aggressiveness or metastatic changes. Finally, the most intensive changes in microbiota relative to location were seen in peritumoural tissue, possibly reflecting changes in tumoural ECM. Our findings are the first to give essential characteristics and differences within lung microbiota of NSCLC and their susceptibility to tumour lobe location, proposing several possible implications of microbiota in pathology of lung cancer and suggesting potential research directions for better understanding of the lung microbiota-cancer interaction.

\section{Supplementary information}

Supplementary information accompanies this paper at https://doi.org/10. 1186/s12931-020-01392-2.

Additional file 1: Figure 1. Most abundant genera in four lung and salivary microbiota relative to tumour lobe location. Text 1. Chapter "Additional explanation of differences between lung samples (main manuscript: Fig. 6)" that adds details on significant differences found in Fig. 6.

\section{Abbreviations}

ADC: Adenocarcinoma; BAL: Bronchoalveolar lavage fluid; BH: BenjaminiHochberg; BMI: Body mass index; COPD: Chronic obstructive pulmonary disease; DLCO: Predicted diffusing capacity of the lung for carbon monoxide; ECM: Extracellular matrix; FEV 1 : Forced expiratory volume per second; FVC: Forced vital capacity; KW: Kruskal-Wallis test; LC: Lung cancer; LL: Lower lobe; LUNG.DP: Non-malignant distal lung piece; LUNG.PT: Peritumoural lung tissue; LUNG.T: Tumour tissue; NMDS: Non-metric multidimensional scaling; NSCLC: Non-small cell lung cancer; OTU: Outer taxonomic unit; PD1: Programmed cell Death protein 1; QIIME: Quantitative Insights Into Microbial Ecology; SCC: Squamous cell carcinoma; T: Tumour; UL: Upper lobe; uwUF: Unweighted UniFranc distance; wUF: Weighted UniFrac distance

\section{Acknowledgements}

The authors thank all the patients who accepted participation in this protocol. They gratefully acknowledge clinical research associates, Margaux Marcoux and Emilie Thivat, for managing administrative aspects of the study. The authors gratefully acknowledge the help of Laureen Crouzet and Floriane Serre (UMR0454 MEDIS, INRA-UCA) in optimisation of the DNA extraction protocol for lung microbiota and for technical help in DNA isolation, respectively.

\section{Data sharing}

This statement is intended to clarify Centre Jean Perrin's position on data sharing with regards to applicable regulatory frameworks.

\section{European regulatory framework}

De-identified study data are considered as personal data within the meaning of Regulation (EU) 2016/679 of the European Parliament and of the Council of 27 April 2016, known as « GDPR ».

As a result, their transfer places further processors in the scope of GDPR (Art.

2 and 3 ) and is notably subject to

- Precise information of study subjects (Art. 14),

- Prior identification of further processors and signature of a transfer

agreement (Chapter V),

- Data protection impact assessment (Art. 35).

\section{French regulatory framework}

In addition to GDPR, the transfer of this data by Centre Jean Perrin is a processing in itself that is subject to Chapters IX and XII of the updated Law no. 78-17 of 6 January 1978. Within the framework of this law, the data processing is permitted by Centre Jean Perrin's conformity to the MR-001 Code of conduct ("Délibération n²018-153 du 3 mai 2018 portant homologation 
d'une méthodologie de référence relative aux traitements de données à caractère personnel mis en œuvre dans le cadre des recherches dans le domaine de la santé avec recueil du consentement de la personne concernée").

In its last update from May 2018, MR-001 was updated to allow transfer to reviewers within certain conditions (Article 2.4)

These conditions can be summarised as follow:

The access to study data by an independent mandated reviewer can only be granted through an interface chosen by the data processor (i.e. Centre Jean Perrin, in this context) for the sole purpose of re-analysis or for any other purpose supported by a strong rational and a project synopsis describing why access to this study's data (study acronym: MICA) is necessary. The data processor shall ensure:

- That the chosen sharing platform cannot allow extraction of the data; - To grant specific personal and differentiated authorisations to access the data;

- That the users be reliably identified

- That state-of-the-art cryptography and security measures are used;

- That an audit trail of accesses is used:

- When data is transferred outside of the EU, that applicable transfer contracts are established;

- That study subjects are informed of the data recipients;

- That the data is used for the sole purpose of reproducing published results; - That the data is cleaned from any directly identifying data and that the principle of data minimisation is applied (i.e. limiting the transfer to data used in the publication).

\section{Study data sharing}

As a result, for this trial (acronym: MICA), Centre Jean Perrin can share individual participant data that underlie the results reported in the article under the following conditions:

- Limitation to the data processing to the purpose of independent review of the published results;

- Prior minimisation of the data (i.e. restriction to data strictly adequate, relevant and limited to the purpose of the independent review of results); - Prior identification of the reviewer and authorisation from Centre Jean Perrin for a personal access;

- Following the signature of a transfer agreement with Centre Jean Perrin, in conformity with the European Commission's Standard Contractual Clauses; Access to study data will be assessed, upon written detailed request sent to the project manager Emilie THIVAT: emilie.thivat@clermont.unicancer.fr, from 6 months until 5 years following article's publication.

\section{Authors' contributions}

$A B D, E F, J Y B, M F$ and MPV were responsible for the study design. ABD, ED, MF and RB were involved in the protocol development and optimisation. MF and RB planned and performed the experiments. RB analysed the data with the help from IM for the statistical approach. MF and ABD aided in interpreting the results. RB designed the figures and wrote the manuscript. All authors gave their critical feedback for the final manuscript version.

\section{Funding}

This work was financially supported by the Auvergne Region (France) European Regional Development Fund (ERDF) and company Greentech (Saint-Beauzire, France). The study sponsor was Centre Jean Perrin, ClermontFerrand, France.

\section{Availability of data and materials}

According to the practice of the sponsoring institution (Centre Jean Perrin), all samples will be preserved for 15 years. The datasets generated and/or analysed during the current study are confidential but are available from the corresponding author on reasonable request and after the data sharing process is brought into compliance with GDPR (Regulation (EU) 2016/679 of the European Parliament and of the Council of 27 April 2016).

\section{Ethics approval and consent to participate}

The study (and its amendment in June 2018) was approved by the Committee for the Protection of Persons (CPP) Sud-Est VI, Clermont-Ferrand, France, and The French National Agency for Medicines and Health Products Safety (ANSM) (study ref. 2016-A01640-51). The study is registered with the
Clinical Trials under ID: NCT03068663. Written informed consent was obtained from all patients before enrolment in the study.

\section{Consent for publication}

Not applicable.

\section{Competing interests}

The authors declare that they have no competing interests.

\section{Author details}

'Université Clermont Auvergne, INRAE, UNH, F-63000 Clermont-Ferrand, France. ${ }^{2}$ Greentech SA, Biopole Clermont-Limagne, 63360 Saint-Beauzire, France. ${ }^{3}$ Centre Jean Perrin, INSERM, U1240 Imagerie Moléculaire et Stratégies Théranostiques, Université Clermont Auvergne, F-63011 Clermont-Ferrand, France. ${ }^{4}$ Délégation Recherche Clinique \& Innovation, Centre Jean Perrin, Centre de Lutte contre le Cancer, F-63011

Clermont-Ferrand, France. ${ }^{5}$ Centre d'Investigation Clinique, UMR501, F-63001 Clermont-Ferrand, France. ${ }^{6}$ Université Clermont Auvergne, INRAE, MEDIS, 63122 Saint-Genes-Champanelle, France. ${ }^{7}$ Centre Jean Perrin, CHU Gabriel-Montpied, Clinical Nutrition Unit, F-63000 Clermont-Ferrand, France. ${ }^{8}$ Thoracic Surgery Department, Centre Jean Perrin, 63011 Clermont-Ferrand, France.

Received: 5 December 2019 Accepted: 10 May 2020

Published online: 08 July 2020

\section{References}

1. Stewart BW, Wild CP. World cancer report 2014. 2014th ed. Lyon: International Agency for Research on Cancer; 2014. doi:9283204298.

2. Goldstraw P, Chansky K, Crowley J, Rami-Porta R, Asamura H, Eberhardt WEE et al. The IASLC lung cancer staging project: proposals for revision of the TNM stage groupings in the forthcoming (eighth) edition of the TNM classification for lung cancer. J Thorac Oncol. 2016;11:39-51. https://doi.org/ 10.1016/j.jtho.2015.09.009.

3. Asamura H, Goya T, Koshiishi Y, Sohara Y, Eguchi K, Mori M, et al. A Japanese lung cancer registry study: prognosis of 13,010 resected lung cancers. J Thorac Oncol. 2008;3:46-52. https://doi.org/10.1097/JTO. ob013e31815e8577.

4. Inoue M, Sawabata N, Takeda S, Ohta M, Ohno Y, Maeda H. Results of surgical intervention for p-stage IIIA (N2) non-small cell lung cancer: acceptable prognosis predicted by complete resection in patients with single N2 disease with primary tumor in the upper lobe. J Thorac Cardiovasc Surg. 2004;127:1100-6. https://doi.org/10.1016/j.jtcvs.2003.09.012.

5. Hayakawa K, Mitsuhashi N, Saito Y, Furuta M, Nakayama Y, Katano S, et al. Impact of tumor extent and location on treatment outcome in patients with stage III non-small cell lung cancer treated with radiation therapy. Jpn J Clin Oncol. 1996:26:221-8. https://doi.org/10.1093/oxfordjournals.jico.a023218.

6. Ou S-HHI, Zell JA, Ziogas A, Anton-Culver H. Prognostic factors for survival of stage I nonsmall cell lung cancer patients: a population-based analysis of 19,702 Stage I patients in the California cancer registry from 1989 to 2003. Cancer. 2007:110:1532-41. https://doi.org/10.1002/cncr.22938.

7. Goldszmid RS, Dzutsev A, Viaud S, Zitvogel L, Restifo NP, Trinchieri G. Microbiota modulation of myeloid cells in cancer therapy. Cancer Immunol Res. 2015:3:103-9. https://doi.org/10.1158/2326-6066.CIR-14-0225.

8. Sivan A, Corrales L, Hubert N, Williams JB, Aquino-Michaels K, Earley ZM, et al. Commensal Bifidobacterium promotes antitumor immunity and facilitates anti-PD-L1 efficacy. Science. 2015;350:1084-9. https://doi.org/10. $1126 /$ science aac4255.

9. Routy B, Le Chatelier E, Derosa L, Duong CPM, Alou MT, Daillère R, et al. Gut microbiome influences efficacy of PD-1-based immunotherapy against epithelial tumors. Science. 2018;359:91-7. https://doi.org/10.1126/science. aan3706.

10. Daillère $R$, Vétizou M, Waldschmitt N, Yamazaki T, Isnard C, Poirier-Colame V, et al. Enterococcus hirae and Barnesiella intestinihominis facilitate cyclophosphamide-induced therapeutic immunomodulatory effects. Immunity. 2016;45:931-43. https://doi.org/10.1016/j.immuni.2016.09.009.

11. Viaud S, Saccheri F, Mignot G, Yamazaki T, Daillère R, Hannani D, et al. The intestinal microbiota modulates the anticancer immune effects of cyclophosphamide. Science. 2013:342:971-6. https://doi.org/10.1126/science. 1240537. 
12. Viaud $S$, Daillère $R$, Boneca $I G$, Lepage $P$, Langella $P$, Chamaillard $M$ et al. Gut microbiome and anticancer immune response: really hot Sh*t! Cell Death Differ. 2015;22:199-214. https://doi.org/10.1038/cdd. 2014.56.

13. Bingula R, Filaire M, Radosevic-Robin N, Bey M, Berthon J-Y, BernalierDonadille A, et al. Desired turbulence? Gut-lung axis, immunity, and lung cancer. J Oncol. 2017;2017:1-15. https://doi.org/10.1155/2017/5035371.

14. Jobin C. Precision medicine using microbiota. Science. 2018;359:32-4. https://doi.org/10.1126/science.aar2946.

15. Dickson RP, Erb-Downward JR, Huffnagle GB. Towards an ecology of the lung: new conceptual models of pulmonary microbiology and pneumonia pathogenesis. Lancet Respir Med. 2014;2:238-46. https://doi.org/10.1016/ S2213-2600(14)70028-1.

16. Marsland BJ, Gollwitzer ES. Host-microorganism interactions in lung diseases. Nat Rev Immunol. 2014;14:827-35. https://doi.org/10.1038/ nri3769.

17. Bernasconi E, Pattaroni C, Koutsokera A, Pison C, Kessler R, Benden C, et al. Airway microbiota determines innate cell inflammatory or tissue remodeling profiles in lung transplantation. Am J Respir Crit Care Med. 2016;194:1252-63. https://doi.org/10.1164/rccm.201512-2424OC.

18. Blainey PC, Milla CE, Cornfield DN, Quake SR. Quantitative analysis of the human airway microbial ecology reveals a pervasive signature for cystic fibrosis. Sci Transl Med. 2012;4:153ra130. https://doi.org/10.1126/ scitranslmed.3004458.

19. Dickson RP, Erb-Downward JR, Freeman CM, McCloskey L, Beck JM, Huffnagle GB, et al. Spatial variation in the healthy human lung microbiome and the adapted island model of lung biogeography. Ann Am Thorac Soc. 2015;12:821-30. https://doi.org/10.1513/AnnalsATS.201501-0290C.

20. Dickson RP, Martinez FJ, Huffnagle GB. The role of the microbiome in exacerbations of chronic lung diseases. Lancet. 2014;384:691-702. https:// doi.org/10.1016/S0140-6736(14)61136-3.

21. Sze MA, Dimitriu PA, Hayashi S, Elliott WM, McDonough JE, Gosselink JV, et al. The lung tissue microbiome in chronic obstructive pulmonary disease. Am J Respir Crit Care Med. 2012;185:1073-80. https://doi.org/10.1164/rccm. 201111-20750C

22. Hilty M, Burke C, Pedro H, Cardenas P, Bush A, Bossley C, et al. Disordered microbial communities in asthmatic airways. PLoS One. 2010;5:e8578. https://doi.org/10.1371/journal.pone.0008578.

23. Jin C, Lagoudas GK, Zhao C, Bullman S, Bhutkar A, Hu B, et al. Commensal microbiota promote lung cancer development via $\gamma \delta$ T cells. Cell. 2019;176: 998-1013. https://doi.org/10.1016/j.cell.2018.12.040.

24. Cheng M, Qian L, Shen GGG, Bian G, Xu T, Xu W, et al. Microbiota modulate tumoral immune surveillance in lung through a $\gamma \delta \mathrm{T} 17$ immune celldependent mechanism. Cancer Res. 2014;74:4030-41. https://doi.org/10. 1158/0008-5472.CAN-13-2462.

25. Boursi B, Mamtani R, Haynes K, Yang Y-X. Recurrent antibiotic exposure may promote cancer formation - another step in understanding the role of the human microbiota? Eur J Cancer. 2015;51:2655-64. https://doi.org/10.1016/j. ejca.2015.08.015.

26. Segal LN, Clemente JC, Tsay J-CJ, Koralov SB, Keller BC, Wu BG, et al. Enrichment of the lung microbiome with oral taxa is associated with lung inflammation of a Th17 phenotype. Nat Microbiol. 2016;1:16031. https://doi. org/10.1038/nmicrobiol.2016.31.

27. Segal LN, Clemente JC, Tsay J-CJ, Koralov SB, Keller BC, Wu BG, et al. Enrichment of lung microbiome with supraglottic taxa is associated with increased pulmonary inflammation. Microbiome. 2013;1:19. https://doi.org/ 10.1186/2049-2618-1-19.

28. Tsay JCJ, Wu BG, Badri MH, Clemente JC, Shen N, Meyn P, et al. Airway microbiota is associated with upregulation of the PI3K pathway in lung cancer. Am J Respir Crit Care Med. 2018;198:1188-98. https://doi.org/10. 1164/rccm.201710-21180C.

29. Wang K, Huang Y, Zhang Z, Liao J, Ding Y, Fang $X$, et al. A preliminary study of microbiota diversity in saliva and bronchoalveolar lavage fluid from patients with primary bronchogenic carcinoma. Med Sci Monit. 2019;25: 2819-34. https://doi.org/10.12659/msm.915332.

30. Liu HX, Tao LL, Zhang J, Zhu YG, Zheng Y, Liu D, et al. Difference of lower airway microbiome in bilateral protected specimen brush between lung cancer patients with unilateral lobar masses and control subjects. Int J Cancer. 2018;142:769-78. https://doi.org/10.1002/ijc.31098.

31. Yan X, Yang M, Liu J, Gao R, Hu J, Li J, et al. Discovery and validation of potential bacterial biomarkers for lung cancer. Am J Cancer Res. 2015;5:
3111-22 http://www.ncbi.nlm.nih.gov/pubmed/26693063. Accessed 25 Sep 2019.

32. Yu G, Gail MH, Consonni D, Carugno M, Humphrys M, Pesatori AC, et al. Characterizing human lung tissue microbiota and its relationship to epidemiological and clinical features. Genome Biol. 2016;17:163. https://doi. org/10.1186/s13059-016-1021-1.

33. Peters BA, Hayes RB, Goparaju C, Reid C, Pass HI, Ahn J. The microbiome in lung cancer tissue and recurrence-free survival. Cancer Epidemiol Biomark Prev. 2019;28:731-40. https://doi.org/10.1158/1055-9965.EPI-18-0966.

34. Bingula R, Filaire $M$, Radosevic-Robin N, Berthon J-Y, Bernalier-Donadille A, Vasson M-P, et al. Characterisation of gut, lung, and upper airways microbiota in patients with non-small cell lung carcinoma. Medicine. 2018; 97:e13676. https://doi.org/10.1097/MD.0000000000013676.

35. RStudio Team. RStudio: integrated development environment for R. 2016. http://www.rstudio.com/.

36. McMurdie PJ, Holmes S. Phyloseq: an R package for reproducible interactive analysis and graphics of microbiome census data. PLoS One. 2013;8:e61217. https://doi.org/10.1371/journal.pone.0061217.

37. Oksanen J, Blanchet FG, Friendly M, Kindt R, Legendre P, Mcglinn D, et al. vegan: Community ecology package. 2019. https://cran.r-project.org/web/ packages/vegan/vegan.pdf. Accessed 6 Jun 2019.

38. Lahti L, Shetty S, Blake T, Salojarvi J. microbiome R package. 2017. http:// microbiome.github.io.

39. Wickham H. ggplot2: Elegant graphics for data analysis. 2016. http:// ggplot2.org.

40. Love MI, Huber W, Anders S. Moderated estimation of fold change and dispersion for RNA-seq data with DESeq2. Genome Biol. 2014;15:550. https://doi.org/10.1186/s13059-014-0550-8.

41. Foster ZSL, Sharpton TJ, Grünwald NJ. Metacoder: an R package for visualization and manipulation of community taxonomic diversity data. PLoS Comput Biol. 2017;13:e1005404. https://doi.org/10.1371/journal.pcbi.1005404

42. Dickson RP, Cox MJ. Gut microbiota and protection from pneumococcal pneumonia. Gut. 2017:66:384. https://doi.org/10.1136/gutjnl-2016-311823.

43. Sze MA, Hogg JC, Sin DD. Bacterial microbiome of lungs in COPD. Int J COPD. 2014;9:229-38. https://doi.org/10.2147/COPD.S38932.

44. Vital M, Harkema JR, Rizzo M, Tiedje J, Brandenberger C. Alterations of the murine gut microbiome with age and allergic airway disease. J Immunol Res. 2015;2015:1-8. https://doi.org/10.1155/2015/892568.

45. Dickson RP, Erb-Downward JR, Freeman CM, Walker N, Scales BS, Beck JM, et al. Changes in the lung microbiome following lung transplantation include the emergence of two distinct Pseudomonas species with distinct clinical associations. PLoS One. 2014;9:e97214. https://doi.org/10.1371/ journal.pone.0097214.

46. Fodor AA, Klem ER, Gilpin DF, Elborn JS, Boucher RC, Tunney MM, et al. The adult cystic fibrosis airway microbiota is stable over time and infection type, and highly resilient to antibiotic treatment of exacerbations. PLoS One. 2012;7:e45001. https://doi.org/10.1371/journal.pone.0045001.

47. Lee SH, Sung JY, Yong D, Chun J, Kim SKSSY, Song JH, et al. Characterization of microbiome in bronchoalveolar lavage fluid of patients with lung cancer comparing with benign mass like lesions. Lung Cancer. 2016;102:89-95. https://doi.org/10.1016/j.lungcan.2016.10.016.

48. Dickson RP, Erb-downward JR, Prescott HC, Martinez FJ, Curtis JL, Lama VN, et al. Cell-associated bacteria in the human lung microbiome. Microbiome. 2014;2:1-10. https://doi.org/10.1186/2049-2618-2-28.

49. Snoeckx A, Dendooven A, Carp L, Desbuquoit D, Spinhoven MJ, Lauwers $P$, et al. Wolf in sheep's clothing: primary lung cancer mimicking benign entities. Lung Cancer. 2017;112:109-17. https://doi.org/10.1016/j.lungcan. 2017.07.037.

50. Busch SE, Hanke ML, Kargl J, Metz HE, MacPherson D, Houghton AM. Lung cancer subtypes generate unique immune responses. J Immunol. 2016;197: 4493-503. https://doi.org/10.4049/jimmunol.1600576.

51. Valkenburg KC, De Groot AE, Pienta KJ. Targeting the tumour stroma to improve cancer therapy. Nat Rev Clin Oncol. 2018;15:366-81. https://doi.org/ 10.1038/s41571-018-0007-1.

52. Charlson ES, Bittinger $K$, Chen J, Diamond JM, Li H, Collman RG, et al. Assessing bacterial populations in the lung by replicate analysis of samples from the upper and lower respiratory tracts. PLoS One. 2012;7:e42786. https://doi.org/10.1371/journal.pone.0042786.

53. Grassl N, Kulak NA, Pichler G, Geyer PE, Jung J, Schubert S, et al. Ultra-deep and quantitative saliva proteome reveals dynamics of the oral microbiome. Genome Med. 2016:8:44. https://doi.org/10.1186/s13073-016-0293-0. 
54. Bassis CM, Erb-Downward JR, Dickson RP, Freeman CM, Schmidt TM, Young $\mathrm{VB}$, et al. Analysis of the upper respiratory tract microbiotas as the source of the lung and gastric microbiotas in healthy individuals. MBio. 2015;6:1-10. https://doi.org/10.1128/mBio.00037-15.

55. Gleeson K, Eggli DF, Maxwell SL. Quantitative aspiration during sleep in normal subjects. Chest. 1997;111:1266-72. https://doi.org/10.1378/chest.111. 5.1266.

56. Anesti V, McDonald IR, Ramaswamy M, Wade WG, Kelly DP, Wood AP. Isolation and molecular detection of methylotrophic bacteria occurring in the human mouth. Environ Microbiol. 2005;7:1227-38. https://doi.org/10. 1111/j.1462-2920.2005.00805.x.

57. Jamieson WD, Pehl MJ, Gregory GA, Orwin PM. Coordinated surface activities in Variovorax paradoxus EPS. BMC Microbiol. 2009;9:124. https:// doi.org/10.1186/1471-2180-9-124.

58. Koo H, Allan RN, Howlin RP, Stoodley P, Hall-Stoodley L. Targeting microbial biofilms: current and prospective therapeutic strategies. Nat Rev Microbiol. 2017;15:740-55. https://doi.org/10.1038/nrmicro.2017.99.

59. Riquet M, Pricopi C, Arame A, Barthes FLP. From anatomy to lung cancer: questioning lobe-specific mediastinal lymphadenectomy reliability. J Thorac Dis. 2016;8:2387-90. https://doi.org/10.21037/jtd.2016.08.90.

60. Kotoulas CS, Foroulis CN, Kostikas K, Konstantinou M, Kalkandi P, Dimadi M, et al. Involvement of lymphatic metastatic spread in non-small cell lung cancer accordingly to the primary cancer location. Lung Cancer. 2004;44: 183-91. https://doi.org/10.1016/j.lungcan.2003.10.012.

61. Mukerji R, Kakarala R, Smith SJ, Kusz HG. Chryseobacterium indologenes: an emerging infection in the USA. BMJ Case Rep. 2016;2016:bcr2016214486. https://doi.org/10.1136/bcr-2016-214486.

62. Purty S, Saranathan R, Prashanth K, Narayanan K, Asir J, Sheela Devi C, et al. The expanding spectrum of human infections caused by Kocuria species: a case report and literature review. Emerg Microbes Infect. 2013. https://doi. org/10.1038/emi.2013.71

63. Pragman AA, Kim HB, Reilly CS, Wendt C, Isaacson RE. The lung microbiome in moderate and severe chronic obstructive pulmonary disease. PLoS One. 2012;7:e47305. https://doi.org/10.1371/journal.pone.0047305.

64. Savini V, Gherardi G, Astolfi D, Polilli E, Dicuonzo G, D'Amario C, et al. Insights into airway infections by enterococci: a review. Recent Pat Antiinfect Drug Discov. 2012;7:36-44. https://doi.org/10.2174/ 157489112799829774.

65. Werner JL, Escolero SG, Hewlett JT, Mak TN, Williams BP, Eishi Y, et al. Induction of pulmonary granuloma formation by propionibacterium acnes is regulated by MyD88 and Nox2. Am J Respir Cell Mol Biol. 2017;56:121-30. https://doi.org/10.1165/rcmb.2016-00350C.

66. Henao-Martínez AF, González-Fontal GR, Castillo-Mancilla JR, Yang IV. Enterobacteriaceae bacteremias among cancer patients: an observational cohort study. Int J Infect Dis. 2013;17:e374-8. https://doi.org/10.1016/j.jijd. 2012.11.030.

67. Bhat R, Bissell MJ. Of plasticity and specificity: dialectics of the microenvironment and macroenvironment and the organ phenotype. Wiley Interdiscip Rev Dev Biol. 2014;3:147-63. https://doi.org/10.1002/wdev.130.

68. Quail DF, Joyce JA. Microenvironmental regulation of tumor progression and metastasis. Nat Med. 2013;19:1423-37. https://doi.org/10.1038/nm.3394.

69. Bissell MJ, Hines WC. Why don't we get more cancer? A proposed role of the microenvironment in restraining cancer progression. Nat Med. 2011;17: 320-9. https://doi.org/10.1038/nm.2328.

70. Altinay S. Is extracellular matrix a castle against to invasion of cancer cells? In: Tumor metastasis. London: IntechOpen; 2016. https://doi.org/10.5772/ 64495.

71. Wouters BG, Koritzinsky M, Chiu RK, Theys J, Buijsen J, Lambin P. Modulation of cell death in the tumor microenvironment. Semin Radiat Oncol. 2003;13: 31-41. https://doi.org/10.1053/srao.2003.50004.

72. Fadaka A, Ajiboye B, Ojo O, Adewale O, Olayide I, Emuowhochere R. Biology of glucose metabolization in cancer cells. J Oncol Sci. 2017;3:45-51. https:// doi.org/10.1016/j.jons.2017.06.002.

73. Bagheri R, Haghi SZ, Dalouee MN, Nasiri Z, Rajabnejad A. Evaluation of the results of surgery treatment in patients with benign lung tumors. Lung India. 2015;32:29-33. https://doi.org/10.4103/0970-2113.148436.

74. Khan A, Al-Jahdali H, Irion K, Arabi M, Koteyar S. Solitary pulmonary nodule: a diagnostic algorithm in the light of current imaging technique. Avicenna Med. 2011;1:39-51. https://doi.org/10.4103/2231-0770.90915.

75. Kalchiem-Dekel O, Galvin J, Burke A, Atamas S, Todd N. Interstitial lung disease and pulmonary fibrosis: a practical approach for general medicine physicians with focus on the medical history. J Clin Med. 2018;7:476. https:// doi.org/10.3390/jcm7120476.

76. Luh SP. Diagnosis and treatment of primary spontaneous pneumothorax. J Zhejiang Univ Sci B. 2010;11:735-44. https://doi.org/10.1631/jzus.B1000131.

77. Dimakou K, Chrysikos S, Melachroinidou M, Triantafillidou C. Bronchiectasis and emphysema. Pneumon. 2016;29:334-7.

78. Erb-Downward JR, Thompson DL, Han MK, Freeman CM, McCloskey L, Schmidt LA, et al. Analysis of the lung microbiome in the "healthy" smoker and in COPD. PLoS One. 2011;6:e16384. https://doi.org/10.1371/journal. pone.0016384.

79. Wild CP, Weiderpass E, Stewart BW. World cancer report: cancer research for cancer prevention. 2020th ed. Lyon: International Agency for Research on Cancer; 2020. http://publications.iarc.fr/586.

\section{Publisher's Note}

Springer Nature remains neutral with regard to jurisdictional claims in published maps and institutional affiliations.
Ready to submit your research? Choose BMC and benefit from:

- fast, convenient online submission

- thorough peer review by experienced researchers in your field

- rapid publication on acceptance

- support for research data, including large and complex data types

- gold Open Access which fosters wider collaboration and increased citations

- maximum visibility for your research: over $100 \mathrm{M}$ website views per year

At BMC, research is always in progress.

Learn more biomedcentral.com/submissions 